\title{
ON THE MAXIMAL PARAMETER RANGE OF GLOBAL STABILITY FOR A NONLOCAL THERMOSTAT MODEL
}

\author{
PATRICK GUIDOTTI AND SANDRO MERINO
}

\begin{abstract}
The global asymptotic stability of the unique steady state of a nonlinear scalar parabolic equation with a nonlocal boundary condition is studied. The equation describes the evolution of the temperature profile that is subject to a feedback control loop. It can be viewed as a model of a rudimentary thermostat, where a parameter controls the intensity of the heat flow in response to the magnitude of the deviation from the reference temperature at a boundary point. The system is known to undergo a Hopf bifurcation when the parameter exceeds a critical value. Results on the characterization of the maximal parameter range where the reference steady state is globally asymptotically stable are obtained by analyzing a closely related nonlinear Volterra integral equation. Its kernel is derived from the trace of a fundamental solution of a linear heat equation. A version of the Popov criterion is adapted and applied to the Volterra integral equation to obtain a sufficient condition for the asymptotic decay of its solutions.
\end{abstract}

\section{INTRODUCTION}

The nonlocal nonlinear problem

$$
\begin{cases}u_{t}-u_{x x}=0 & \text { in }(0, \infty) \times(0, \pi), \\ u_{x}(t, 0)=\tanh (\beta u(t, \pi)) & \text { for } t \in(0, \infty), \\ u_{x}(t, \pi)=0 & \text { for } t \in(0, \infty), \\ u(0, \cdot)=u_{0} & \text { in }(0, \pi),\end{cases}
$$

with parameter $\beta \in \mathbb{R}$ was first introduced in [11] as a simple model for a thermostat where the sensor is not placed in the same position as the heating/cooling actuator that receives temperature feedback. In that paper, it was shown that the local exponential stability of the trivial solution $u(t, \cdot) \equiv 0$ is lost when $\beta \in(0, \infty)$ exceeds the critical value $\beta_{0} \approx 5.6655$. In fact, in [1] it is shown that a Hopf bifurcation occurs at $\beta_{0}$ which produces a local branch of periodic solutions for $\beta \in\left(\beta_{0}, \beta_{0}+\varepsilon\right)$ and some $\varepsilon>0$. In this paper we show that the trivial solution is globally attractive for $\beta \in\left(0, \beta_{0}\right)$. Following the terminology used in [17] we need to distinguish between the concept of the attractor $\hat{\mathcal{A}}_{\beta}$ and the B-attractor $\mathcal{A}_{\beta}$ when formulating our main results in Theorem 5.1. We point out that, in general, the attractor $\hat{\mathcal{A}}_{\beta}$ is a proper subset of the B-attractor $\mathcal{A}_{\beta}$. We prove that the continuous semiflow $\Phi_{\beta}$ induced on $\mathrm{H}^{1}(0, \pi)$ by the system $(1.1)$ has a global attractor $\hat{\mathcal{A}}_{\beta}=\{0\}$ for $\beta \in\left(0, \beta_{0}\right)$ and that for $\beta \in\left(0, \frac{4}{\pi}\right)$ the B-attractor and the attractor coincide, i.e. $\mathcal{A}_{\beta}=\hat{\mathcal{A}}_{\beta}=\{0\}$. In [7], the authors prove that the global B-attractor $\mathcal{A}_{\beta}$ exists for $\beta \in(0, \infty)$ and that $\mathcal{A}_{\beta}=\{0\}$ for $\beta \in\left(0, \frac{1}{\pi}\right)$. We thus extend previous results by determining larger parameter ranges where the B-attractor and the attractor are shown to be equal to $\{0\}$.

The existence of the B-attractor $\mathcal{A}_{\beta}$ shown in [7] for $\beta \in(0, \infty)$ implies, in particular, that all orbits are bounded in the underlying Banach space. Since $\mathrm{H}^{1}(0, \pi) \hookrightarrow \mathrm{C}([0, \pi])$, the orbits satisfy

$$
\left\|\Phi_{\beta}\left(t, u_{0}\right)\right\|_{\infty} \leq c\left(u_{0}\right)<\infty, t \geq 0 .
$$

Key words and phrases. nonlinear reaction diffusion systems, nonlocal boundary conditions, nonlinear feedback control systems, Popov criterion, Volterra integral equation, global attractor. 
By means of a weak formulation we interpret $(1.1)$ as the abstract Cauchy problem

$$
\left\{\begin{array}{l}
\dot{u}+A_{N} u=-\gamma_{0}^{\prime}\left(\tanh \left(\beta \gamma_{\pi} u(t)\right)\right), \quad t>0, \\
u(0)=u_{0}
\end{array}\right.
$$

in the Banach space $\mathrm{H}^{-1}=\left(\mathrm{H}^{1}(0, \pi)\right)^{\prime}$, where $\mathrm{H}^{-1}$ is the dual space of $\mathrm{H}^{1}:=\mathrm{H}^{1}(0, \pi)$. Here $A_{N}$ denotes the unbounded operator

$$
A_{N}: \operatorname{dom}\left(A_{N}\right)=\mathrm{H}^{1} \subset \mathrm{H}^{-1} \rightarrow \mathrm{H}^{-1}
$$

given by

$$
\left\langle A_{N} u, v\right\rangle=\int_{0}^{\pi} u_{x} v_{x} d x, u, v \in \mathrm{H}^{1},
$$

and the linear operators $\gamma_{\pi} \in \mathcal{L}\left(\mathrm{H}^{1}, \mathbb{R}\right)$ with $\gamma_{\pi}(u)=u(\pi)$ and $\gamma_{0}^{\prime} \in \mathcal{L}\left(\mathbb{R}, \mathrm{H}^{-1}\right)$ with $\left\langle\gamma_{0}^{\prime}(s), v\right\rangle=s \gamma_{0}(v)$, for $s \in \mathbb{R}$ and $v \in \mathrm{H}^{1}$ denote the trace operator at $x=\pi$ and the dual of the trace operator at $x=0$, respectively. Notice, in particular, that $\gamma_{0}^{\prime}(s)=s \delta_{0}$, where $\delta_{0} \in \mathrm{H}^{-1}$ is the Dirac distribution supported in $x=0$, i.e. $\delta_{0}$ is defined by the duality pairing $\left\langle\delta_{0}, \varphi\right\rangle_{\mathrm{H}^{-1}, \mathrm{H}^{1}}:=\varphi(0)$.

The solution of 1.2 exists globally and can be represented by the variation of constants formula

$$
\begin{aligned}
u\left(t, u_{0}\right) & =e^{-t A_{N}} u_{0}-\int_{0}^{t} e^{-(t-\tau) A_{N}} \gamma_{0}^{\prime}\left[\tanh \left(\beta \gamma_{\pi}\left(u\left(\tau, u_{0}\right)\right)\right)\right] d \tau \\
& =e^{-t A_{N}} u_{0}-\int_{0}^{t} \tanh \left[\beta \gamma_{\pi}\left(u\left(\tau, u_{0}\right)\right)\right] e^{-(t-\tau) A_{N}} \delta_{0} d \tau
\end{aligned}
$$

where the second identity holds due to the linearity of the semigroup $\left\{e^{-t A_{N}} \mid t \geq 0\right\}$ generated by the operator $-A_{N}$ on $\mathrm{H}^{-1}$, which is analytic and strongly continuous. As described in [11] and [12] the general theory presented in [1] allows one to formulate the Cauchy Problem (1.2) on a scale of Banach spaces which includes e.g. $\mathrm{H}^{1} \subset \mathrm{H}^{-1}$. As discussed in [11], and further explored in [7], the global semiflow $\Phi_{\beta}$ induced by (1.2) consists of classical solutions $u\left(t, u_{0}\right)(x)$ of the parabolic initial boundary value problem (1.1).

We conclude this section with a brief and incomplete overview on the research that the seemingly innocuous thermostat model triggered since its introduction in [11. In that paper a brief passage in N. Wiener's book "Cybernetics" is quoted and indicated as the source of initial inspiration.

Results about the linear(ized) model have been discussed in [15] also allowing the sensor location to be any $x \in(0, \pi]$. They follow an approach based on an integral reformulation of the problem via the Laplace transform. They observe that the same phenomena rigorously proven in [11] remain valid if the sensor is placed in a different location than the actuator, no matter how close they may be. Global stability of the trivial solution has been studied in [7], where the authors prove the existence of a bounded global attractor $\mathcal{A}_{\beta}$ for any $\beta>0$ and manage to obtain global stability of the trivial solution for $\beta \in\left(0, \frac{1}{\pi}\right)$, showing that $\mathcal{A}_{\beta}=\{0\}$ in that parameter range. In Remarks 1.1 and 1.2 , we give two simple proofs for global stability. The first recovers the global stability result for $\beta \in\left(0, \frac{1}{\pi}\right)$ and the second extends the parameter range of global stability to $\left(0, \frac{4}{\pi}\right)$.

Problem (1.1) has also been studied in the presence of noise in [19]. There the author shows that the average solution of the thermostat problem with randomly switching locations of the sensor and of the actuator can exhibit exponential growth in spite of the fact that stability holds for both configurations.

The observation that the first eigenfunction of the linearization of the stationary problem remains positive up to $\beta=\frac{1}{2}$ and that, for $\beta \in\left(0, \frac{1}{2}\right)$, the semi-group is non-positive leads to the concept of eventually positive semi-groups. These ideas have recently been introduced and developed in [6, 5].

Various nonlinear stationary problems associated with (1.1) have been studied by several authors. We only mention G. Infante and J.R.L Webb ([13], [14]) here and refer to their bibliography for numerous additional results. 
The Hopf bifurcation phenomenon engendered by the nonlocal nature of the boundary condition has also inspired the research presented in [10] on a market price formation model introduced by J.M. Lasry and P.L. Lions. In particular, in that specific context a similar Hopf bifurcation scenario shows that "demand" and "supply" do not simply create stable prices but can lead to price oscillations. The phenomenon emerges on the basis of the modelled behaviour of the population densities of buyers and sellers positioned in a liquid market over a continuum of prospective transaction prices.

The variety of results obtained since the introduction of this rudimentary thermostat model for the original purpose of underpinning N. Wiener's postulated "wild oscillations" more explicitly and rigorously, bears witness to the interesting underlying mathematical properties of this prototypical "parabolic oscillator".

Before proceeding to the derivation of our main result in the next section, we provide two simpler proofs, albeit at the cost of only obtaining smaller ranges for global stability. In the first remark it is shown that the stability result of [7] can be obtained in a different way that gives additional insight into the behavior of solutions. In Remark 1.2 we provide a sharper proof that extends the stability range from $\left(0, \frac{1}{\pi}\right)$ to $\left(0, \frac{4}{\pi}\right)$.

Remark 1.1. A simple calculation shows that

$$
\frac{1}{2} \frac{d}{d t} \int_{0}^{\pi} u^{2}(t, \xi) d \xi=-\int_{0}^{\pi} u_{x}^{2}(t, \xi) d \xi-u(t, 0) \tanh (\beta u(t, \pi))
$$

so that $\|u(t, \cdot)\|_{2}$ can only grow if $u(t, 0) u(t, \pi)<0$. An application of the Cauchy-Schwarz inequality yields that

It follows that

$$
(u(t, 0)-u(t, \pi))^{2}=\left(\int_{0}^{\pi} u_{x}(t, x) d x\right)^{2} \leq \pi \int_{0}^{\pi} u_{x}^{2}(t, \xi) d \xi
$$

$$
\frac{1}{2} \frac{d}{d t} \int_{0}^{\pi} u^{2}(t, \xi) d \xi \leq-u(t, 0) \tanh (\beta u(t, \pi))-\frac{1}{\pi}(u(t, 0)-u(t, \pi))^{2},
$$

and that $\frac{d}{d t}\|u(t, \cdot)\|_{2}^{2}$ can only be non-negative if

$$
\frac{1}{\pi}(u(t, 0)-u(t, \pi))^{2} \leq \tanh (\beta|u(t, \pi)|)|u(t, 0)| .
$$

Therefore for non-negativity it is necessary that

$$
\frac{1}{\pi}|u(t, 0)| \leq \tanh (\beta|u(t, \pi)|),
$$

i.e., that $|u(t, 0)| \leq \pi$, and that

$$
\frac{1}{\pi}|u(t, \pi)|^{2} \leq \tanh (\beta|u(t, \pi)|)|u(t, 0)| \leq \pi,
$$

i.e., that $|u(t, \pi)| \leq \pi$, but also that

$$
|u(t, \pi)|^{2} \leq \pi^{2} \tanh ^{2}(\beta|u(t, \pi)|),
$$

which entails that

$$
|u(t, \pi)| \leq \pi \tanh (\beta|u(t, \pi)|) \leq \pi \beta|u(t, \pi)|,
$$

or, equivalently, that $\beta \geq \frac{1}{\pi}$. This yields the stability of the trivial solution for $\beta \in\left(0, \frac{1}{\pi}\right)$ but also the boundedness of the orbits. We don't give the details of the argument and refer to the result on the existence of the global B-attractor for any $\beta>0$ which is proven in [7] and motivated us to revisit this problem.

In the next remark we extend the stability range to $\beta \in\left(0, \frac{4}{\pi}\right)$ by a relatively simple and direct argument. 
Remark 1.2. Rewrite 1.2 as

$$
u_{t}+A_{N} u=-\frac{\tanh (\beta z)}{z} \gamma_{\pi}(u) \delta_{0}
$$

for $z(t)=\gamma_{\pi}(u(t, \cdot))$. Notice that $\psi(z)(t)=\frac{\tanh (\beta z(t))}{z(t)} \in[\delta, \beta]$ for $t \geq 0$ and some $\delta=\delta\left(u_{0}\right)>0$ since solutions of 1.2 remain pointwise bounded as shown in [7]. We claim that

$$
\frac{1}{2} \int_{0}^{\pi} u^{2}(x) d x=\frac{1}{2}(u, u)_{2}
$$

is a Ljapunov functional for the equation as long as $\beta \in\left(0, \frac{4}{\pi}\right)$. In Section 5 we will discuss that this implies that the $B$-attractor is given by $A_{\beta}=\{0\}$ for that range of the parameter $\beta$. In order to verify that we indeed have a Ljapunov functional we compute

$$
\begin{aligned}
\frac{d}{d t} \frac{1}{2}(u, u)_{2} & =\left\langle-A_{N} u-\psi(z) u(\cdot, \pi) \delta_{0}, u\right\rangle_{\mathrm{H}^{-1}, \mathrm{H}^{1}} \\
& =-\left\langle A_{N} u, u\right\rangle_{\mathrm{H}^{-1}, \mathrm{H}^{1}}-\psi(z) u(\cdot, \pi) u(\cdot, 0) \\
& =-\left\langle A_{N} u, u\right\rangle_{\mathrm{H}^{-1}, \mathrm{H}^{1}}-\psi(z)\left\langle\frac{1}{2}\left(\delta_{0} \delta_{\pi}^{\top}+\delta_{\pi} \delta_{0}^{\top}\right) u, u\right\rangle_{\mathrm{H}^{-1}, \mathrm{H}^{1}} \\
& =-\left\langle A_{N} u+\frac{\psi(z)}{2}\left(\delta_{0} \delta_{\pi}^{\top}+\delta_{\pi} \delta_{0}^{\top}\right) u, u\right\rangle_{\mathrm{H}^{-1}, \mathrm{H}^{1}},
\end{aligned}
$$

where we use the suggestive notation $\delta_{x}^{\top}:=\gamma_{x}$ for $x \in\{0, \pi\}$ to highlight the inherent symmetry. Thus we conclude that the orbital derivative is strictly negative on non-stationary orbits if the self-adjoint operators

$$
A_{N}+\frac{\alpha}{2}\left(\delta_{0} \delta_{\pi}^{\top}+\delta_{\pi} \delta_{0}^{\top}\right)
$$

only possess strictly positive eigenvalues for any parameter value $\alpha \in(\delta, \beta)$. We will now prove that this is the case for $\alpha \in\left(0, \frac{4}{\pi}\right)$. The operators in (1.4) correspond to the weak formulation of the homogeneous heat equation in $(0, \pi)$ with boundary conditions

$$
u_{x}(t, 0)=\frac{\alpha}{2} u(t, \pi), u_{x}(t, \pi)=-\frac{\alpha}{2} u(t, 0) \text { for } t>0 .
$$

The eigenfunctions to a putative eigenvalue $\lambda^{2} \in \mathbb{R}$ can be assumed to be of the form $A \sin (\lambda x)+$ $B \cos (\lambda x)$. In fact, the trace operators added to the Neumann Laplacian in (1.4) constitute a compact perturbation, as is discussed in [12. Therefore the operators (1.4) conserve a pure point spectrum. Imposing the boundary conditions leads to a linear system that only admits non-trivial solutions if its determinant vanishes. The characteristic equation is easily found to be

$$
\lambda-\left[\frac{\alpha^{2}}{4}+\lambda^{2}\right] \frac{\sin (\lambda \pi)}{\alpha}=0 .
$$

If negative eigenvalues $\lambda^{2}$ exist, then they need to be of the form $\lambda=i \mu$ for $\mu \in \mathbb{R}$. Then $\mu$ is a solution of

$$
\mu=\left[\frac{\alpha^{2}}{4}-\mu^{2}\right] \frac{\sinh (\mu \pi)}{\alpha}=: r_{\alpha}(\mu) .
$$

Note that for $|\mu|>\frac{\alpha}{2}, \mathrm{id}_{\mathbb{R}}$ and $r_{\alpha}$ have opposite signs. Any non-trivial solution is therefore confined to the interval $\left[-\frac{\alpha}{2}, \frac{\alpha}{2}\right]$, and, owing to the oddity of the functions, we only need to examine the positive half of that interval. A simple calculation shows that $r_{\alpha}^{\prime}(0)=\frac{\pi}{4} \alpha$, so that $r_{\alpha}^{\prime}(0)<1$ as long as $\alpha<\frac{4}{\pi}$. We proceed to show that $r_{\alpha}$ is strictly concave in $\left[0, \frac{\alpha}{2}\right]$ for $\alpha \in\left(0, \frac{4}{\pi}\right)$. As a consequence no solution $\mu$ of the characterisitic equation can exist and hence no non-positive eigenvalue of (1.4) is found for $\alpha \in\left(0, \frac{4}{\pi}\right)$. The straightforward computation of the second derivative of $r_{\alpha}$ yields

$$
4 \alpha r_{\alpha}^{\prime \prime}(\mu)=\sinh (\mu \pi)\left[\pi^{2}\left(\alpha^{2}-4 \mu^{2}\right)-8\right]-16 \pi \mu \cosh (\mu \pi) .
$$


To find a criterion that implies a negative second derivative of $r_{\alpha}$ observe that

$$
\frac{\tanh (\mu \pi)}{\mu}\left[\pi^{2}\left(\alpha^{2}-4 \mu^{2}\right)-8\right] \leq \pi\left[\pi^{2}\left(\alpha^{2}-4 \mu^{2}\right)-8\right] \leq \alpha^{2} \pi^{3}-8 \pi .
$$

This leads to the condition $\alpha^{2} \pi^{3}-8 \pi<16 \pi$ which holds when $\alpha$ is less than $\frac{\sqrt{24}}{\pi}>\frac{4}{\pi}$. The desired concavity follows and we conclude that the operators (1.4) have a strictly positive point spectrum, whenever $\alpha \in\left(0, \frac{4}{\pi}\right)$. To see that this construction of the Ljapunov functional cannot be extended indefinitely, note that, for $\alpha>\frac{4}{\pi}, r_{\alpha}^{\prime}(0)>1$, which together with $r_{\alpha}\left(\frac{\alpha}{2}\right)=0$ implies that the characterisitic equation $\mu=r_{\alpha}(\mu)$ has at least one solution in $\left[0, \frac{\alpha}{2}\right]$ thus producing a negative eigenvalue of (1.4).

In this paper a different approach is presented that will ensure stability of the trivial solution all the way to the Hopf bifurcation from the steady state that occurs for $\beta=\beta_{0} \approx 5.6655$. The proof will not construct a Ljapunov functional explicitly. It sharpens the result in this remark to a maximal range of stability, yet at the expense of a significantly more technical proof.

\section{The Volterra Integral Equation at the boundary component $\{x=\pi\}$}

The (abstract) variation of constant formula representation (1.3) for the classical solutions of (1.1) can be evaluated at $x=\pi$ to verify that for any given initial state $u_{0} \in \mathrm{H}^{1}$

$$
u(t):=\gamma_{\pi}\left(u\left(t, u_{0}\right)\right),
$$

solves a Volterra integral equation of the second kind. We collect this observation in the next lemma.

Lemma 2.1. Let $\beta \in(0, \infty)$ and let $\Phi_{\beta}\left(\cdot, u_{0}\right)$ be any orbit of the continuous semiflow $\left(\Phi_{\beta}, \mathrm{H}^{1}\right)$ then

$$
u(t):=\gamma_{\pi}\left(\Phi_{\beta}\left(t, u_{0}\right)\right)=\Phi_{\beta}\left(t, u_{0}\right)(x=\pi)
$$

solves the nonlinear convolution-kernel Volterra integral equation of the second kind

$$
y(t)=f(t)+\int_{0}^{t} a(t-\tau) g_{\beta}(y(\tau)) d \tau, t>0
$$

where the so-called forcing function $f \equiv f\left(u_{0}\right)$, the convolution kernel $a$, and $g_{\beta}$ are defined as

$$
\begin{aligned}
f(t) & :=\left(e^{-t A_{N}} u_{0}\right)(\pi) \text { for } t \geq 0, \\
a(t) & :=-\left(e^{-t A_{N}} \delta_{0}\right)(\pi) \text { for } t>0, \\
g_{\beta}(w) & :=\tanh (\beta w) \text { for } w \in \mathbb{R} .
\end{aligned}
$$

Proof. Note that for $t>0$ the analyticity of the semigroup implies that $e^{-t A_{N}} \delta_{0} \in \mathrm{H}^{1}$ since $\delta_{0} \in \mathrm{H}^{-1}$. Hence, thanks to the embedding $\mathrm{H}^{1} \hookrightarrow \mathrm{C}([0, \pi])$, the kernel $a$ can be defined pointwise by evaluating at $x=\pi$. The evaluation at $x=\pi$ for $t>0$ yields

$$
u\left(t, u_{0}\right)(x=\pi)=\left(e^{-t A_{N}} u_{0}\right)(x=\pi)-\int_{0}^{t} \tanh \left[\beta \gamma_{\pi}\left(u\left(\tau, u_{0}\right)\right)\right]\left(e^{-(t-\tau) A_{N}} \delta_{0}\right)(x=\pi) d \tau
$$

and concludes the proof.

Next we collect some remarks, which will be needed in the subsequent study of the above Volterra integral equation.

Remarks 2.2. (a) A full spectral resolution of the operator $A_{N}$ can be computed in order to obtain a series representations for the (kernel of the) semigroup generated by $-A_{N}$, which reads

$$
N(t, x):=e^{-t A_{N}} \delta_{0}=\sum_{k=0}^{\infty} c_{k} \cos (k x) e^{-t k^{2}}
$$


and is the fundamental solution for the parabolic homogeneous Neumann problem on the interval $[0, \pi]$, where $c_{0}=\frac{1}{\pi}$ and $c_{k}=\frac{2}{\pi}$ for $k \geq 1$. Consequently, the integral kernel a satisifies $a=-N(t, \pi)$, i.e. it holds that

$$
a(t)=-\frac{1}{\pi}+\frac{2}{\pi} \sum_{k=1}^{\infty}(-1)^{k+1} e^{-t k^{2}}, t>0 .
$$

Besides the convolution kernel a, the forcing term $f$ can also be expressed in terms of the basis of eigenfunctions to give

$$
f(t)=\sum_{k=0}^{\infty}\left\langle u_{0}, \varphi_{k}\right\rangle e^{-t k^{2}} \varphi_{k}(\pi)=\bar{u}_{0}+\sqrt{\frac{2}{\pi}} \sum_{k=1}^{\infty}(-1)^{k} \hat{u}_{0 k} e^{-t k^{2}},
$$

for $\hat{u}_{0 k}=\left\langle u_{0}, \varphi_{k}\right\rangle$ and $k \in \mathbb{N}$.

(b) For $u_{0} \in \mathrm{H}^{1}$ the forcing function is in $\mathrm{BUC}^{\infty}((0, \infty))$ and its $n$-th derivative satisfies

$$
f^{(n)} \in \mathrm{L}^{p}((0, \infty)) \text { for } p \in[1, \infty], n \geq 1 .
$$

This follows from (2.4), i.e. from the exponential convergence of $u_{N}\left(t, u_{0}\right)$ to the constant function $\bar{u}_{0}$ as $t \rightarrow \infty$.

(c) The fundamental solution (2.2) can also be obtained from the heat kernel on the whole real line:

$$
H(t, x):=\frac{1}{\sqrt{4 \pi t}} e^{-\frac{x^{2}}{4 t}}, x \in \mathbb{R}, t>0 .
$$

First one obtains the kernel $H_{\pi}$ for the $2 \pi$-periodic heat equation by "periodization"

$$
H_{\pi}(t, x):=\sum_{k \in \mathbb{Z}} H(t, x-2 \pi k), x \in[0,2 \pi) .
$$

Then by introducing the Riemann theta function

$$
\theta_{1}(\tau, z):=\sum_{k \in \mathbb{Z}} e^{\pi i k^{2} \tau} e^{2 \pi i k z}, \tau, z \in \mathbb{C}, \operatorname{Im}(\tau)>0
$$

and by setting

$$
\theta(t, x):=\theta_{1}\left(\frac{i t}{\pi}, \frac{x}{2 \pi}\right)=\sum_{k \in \mathbb{Z}} e^{-k^{2} t} e^{i k z}, t>0, x \in \mathbb{R},
$$

it is directly verfied with 2.2 that

$$
N(t, x)=\frac{1}{\pi} \theta(t, x), t>0, x \in[0, \pi] .
$$

It is a classical result (see e.g. [18]) that the periodic heat kernel $H_{\pi}$ can be represented by $\theta$, i.e.

$$
H_{\pi}(t, x)=\frac{1}{2 \pi} \theta(t, x), t>0, x \in[0,2 \pi] .
$$

As a consequence of (2.5) the Neumann fundamental solution $N$ can be written in terms of the periodic heat kernel

$$
N(t, x)=2 H_{\pi}(t, x)=H_{\pi}(t, x)+H_{\pi}(t, 2 \pi-x), t>0, x \in[0, \pi] .
$$

The fundamental solution for the Neumann problem can thus be constructed from the heat kernel on $\mathbb{R}$ by "periodization" and "reflection".

(d) For a and for the "shifted kernel" $a_{s}(t):=a(t)+\frac{1}{\pi}$ it holds that

$$
\lim _{t \rightarrow \infty} a(t)=-\frac{1}{\pi}, \lim _{t \rightarrow \infty} a_{s}(t)=0,
$$

and for $n \geq 1$

$$
\lim _{t \searrow 0} a(t)=\lim _{t \searrow 0} a_{s}^{(n)}(t)=0
$$


Also note that, by definition,

$$
a^{(n)}=a_{s}^{(n)}
$$

for $n \geq 1$. The limits for $t \rightarrow \infty$ are obtained directly from 2.3. To determine the one-sided limits we observe that

$$
-a(t)=N(t, \pi)=2 H_{\pi}(t, \pi)
$$

and therefore the limits for $t \searrow 0$ follow from the well-known properties of the heat kernel on the unit circle. In particular from the concentration of the kernel's mass at $x=0$ as $t \searrow 0$, more precisely

$$
\lim _{t \searrow 0} H_{\pi}(t, x)=\lim _{t \searrow 0} H_{\pi}^{(n)}(t, x)=0, n \geq 1, x \in(0,2 \pi) .
$$

In the sequel we will not need the one-sided limits for the derivatives $a^{(n)}$. For the sake of being more self-contained we also provide an alternative, more direct argument for $\lim _{t \searrow_{0}} a(t)=0$. The Jacobi theta function

$$
\vartheta_{4}(z, q):=\sum_{k=-\infty}^{\infty}(-1)^{k} q^{k^{2}} e^{2 k i z}=1+2 \sum_{k=1}^{\infty}(-1)^{k} q^{k^{2}} \cos (2 k z)
$$

is particularly simple for $z=0$

$$
\vartheta_{4}(q):=\vartheta_{4}(0, q)=1+2 \sum_{k=1}^{\infty}(-1)^{k} q^{k^{2}} .
$$

It is known ( 2], Chapter 3) that $\lim _{q} \jmath_{1} \vartheta_{4}(q)=0$ and therefore

$$
\lim _{q \nearrow 1} \sum_{k=1}^{\infty}(-1)^{k} q^{k^{2}}=-\frac{1}{2} .
$$

Therefore by setting $q=e^{-t}$ we obtain $\lim _{t \searrow_{0}} a(t)=0$ from

$$
\lim _{t \searrow 0} \frac{2}{\pi} \sum_{k=1}^{\infty}(-1)^{k+1} e^{-t k^{2}}=\frac{1}{\pi}
$$

and 2.3 .

(e) The kernel a (extended by 0 for $t \leq 0$ ) satisfies

$$
a \in \mathrm{BC}^{\infty}(\mathbb{R}, \mathbb{R})
$$

and its $n$-th derivatives satisfy

$$
a^{(n)} \in \mathrm{L}^{p}(\mathbb{R}), p \in[1, \infty], n \geq 1 .
$$

This follows from the kernel's representation 2.3 .

(f) Similarly the shifted kernel $a_{s}=a+\frac{1}{\pi}$, extended smoothly to $t=0$ by setting $a_{s}(0)=\frac{1}{\pi}$, satisfies

$$
a_{s} \in \mathrm{BC}^{\infty}([0, \infty), \mathbb{R}) \cap \mathrm{L}^{p}((0, \infty)), p \in[1, \infty] .
$$

Again, this follows from (2.3). Note that the extension of $a_{s}$ to negative arguments by zero creates a discontinuity at $t=0$. This will be relevant when computing its Fourier transform.

(g) The series representation of the Fourier transforms of $a_{s}$ and $a_{s}^{\prime}:=a_{s}^{(1)}$ will be needed in the sequel to recover the Popov stability criterion from the Volterra integral equation. They are given by

$$
\hat{a}_{s}(\omega)=\frac{2}{\pi} \sum_{k=1}^{\infty}(-1)^{k+1} \frac{k^{2}-i \omega}{k^{4}+\omega^{2}}
$$

and by

$$
\widehat{a_{s}^{\prime}}(\omega)=-\frac{1}{\pi}+\frac{2}{\pi} \sum_{k=1}^{\infty}(-1)^{k+1} i \omega \frac{k^{2}-i \omega}{k^{4}+\omega^{2}}
$$


respectively. Note that, here, we define $a_{s}(t)$ also for negative arguments by setting its value to zero for $t<0$. As a consequence of this extension, $a_{s}$ is discontinuous at $t=0$, which impacts the Fourier transform of its distributional derivative $a_{s}^{\prime}$.

To determine the Fourier transforms we use (2.3) and interchange the order of summation and integration, which is justified by the uniform convergence in (2.3). To compute $\hat{a}_{s}$ observe that

$$
\hat{a}_{s}(\omega)=\int_{\mathbb{R}} e^{-i \omega t} a_{s}(t) d t=\int_{0}^{\infty} e^{-i \omega t} a_{s}(t) d t
$$

and therefore

$$
\hat{a}_{s}(\omega)=\frac{2}{\pi} \sum_{k=1}^{\infty}(-1)^{k+1} \int_{0}^{\infty} e^{-i \omega t} e^{-t k^{2}} d t=\frac{2}{\pi} \sum_{k=1}^{\infty}(-1)^{k+1} \int_{0}^{\infty} e^{-\left(i \omega+k^{2}\right) t} d t=\frac{2}{\pi} \sum_{k=1}^{\infty}(-1)^{k+1} \frac{1}{k^{2}+i \omega} .
$$

To find the Fourier transform of $a_{s}^{\prime}$ one can proceed similarly and we evaluate the occuring integral as follows

$$
\begin{aligned}
\widehat{a_{s}^{\prime}}(\omega) & =\int_{0}^{\infty} e^{-i \omega t} a_{s}^{\prime}(t) d t=\lim _{\varepsilon \rightarrow 0}\left\{\frac{2}{\pi} \sum_{k=1}^{\infty}(-1)^{k+2} \int_{\varepsilon}^{\infty} k^{2} e^{-k^{2} t} e^{-i \omega t} d t\right\} \\
& =\lim _{\varepsilon \rightarrow 0}\left\{\left.\frac{2}{\pi} \sum_{k=1}^{\infty}(-1)^{k+1} e^{-k^{2} t} e^{-i \omega t}\right|_{\varepsilon} ^{\infty}\right\}-i \omega \frac{2}{\pi} \sum_{k=1}^{\infty} \int_{0}^{\infty}(-1)^{k} e^{-k^{2} t} e^{-i \omega t} d t .
\end{aligned}
$$

By using (2.7) we find the result

$$
\widehat{a_{s}^{\prime}}(\omega)=-\frac{1}{\pi}+\frac{2}{\pi} \sum_{k=1}^{\infty}(-1)^{k+1} i \omega \frac{\left(k^{2}-i \omega\right)}{k^{4}+\omega^{2}} .
$$

(h) The Laplace transform $\mathcal{L}(a)$ of a is given by

$$
\mathcal{L}(a)(s)=-\frac{1}{\pi s}-\frac{1}{2 \pi} \sum_{k=1}^{\infty}(-1)^{k} \frac{1}{s+k^{2}}, s \in\{z \in \mathbb{C} \mid \operatorname{Re}(z)>0\} .
$$

The Laplace transform $\mathcal{L}(a)$ of the kernel also has the closed form representation

$$
\mathcal{L}(a)(s)=-\frac{1}{\sqrt{s} \sinh (\pi \sqrt{s})} .
$$

The series representation of the Laplace transform is obtained from (2.3) by elementary integrations. Its explicit representation is obtained in [12] (formula (10)) in a different context and is derived again in Section 3.3. A more elementary direct computation using a partial fraction expansion is also given in e.g. [4].

(i) We note that $G_{+}(s):=-\mathcal{L}(a)$ can be expressed in terms of the Fourier transform of $a_{s}$ and that of $a_{s}^{\prime}$. In fact, for $\omega \in \mathbb{R}$, it holds that

$$
\operatorname{Re}\left(G_{+}(i \omega)\right)=-\operatorname{Re}\left(\hat{a}_{s}(\omega)\right)
$$

and

$$
\omega \operatorname{Im}\left(G_{+}(i \omega)\right)=\operatorname{Re}\left(\widehat{a_{s}^{\prime}}(\omega)\right)
$$

The inequality

$$
\operatorname{Re}\left(\hat{a}_{s}(\omega)\right)+q \operatorname{Re}\left(\widehat{a_{s}^{\prime}}(\omega)\right)-\frac{1}{\beta}<0, \omega \in \mathbb{R},
$$

is then equivalent to

$$
\operatorname{Re}\left(G_{+}(i \omega)\right)-q \omega \operatorname{Im}\left(G_{+}(i \omega)\right)>-\frac{1}{\beta}, \omega \in \mathbb{R}
$$


We will use this relationship between $\mathcal{L}(a)$ and $\hat{a}_{s}$ and $\widehat{a_{s}^{\prime}}$ to verify that the stability condition $(2.10)$ obtained from the analysis of the integral equation (2.1) is precisely the Popov stability criterion (2.11) applied to the transfer function $G_{+}$.

The next proposition is one key ingredient for our main stability result. It shows that, in order to infer the decay of all orbits of the semiflow $\left(\Phi_{\beta}, \mathrm{H}^{1}\right)$ to zero in the Banach space $\mathrm{H}^{1}$, it is sufficient to prove that their trace at $x=\pi$ decays to zero in $\mathbb{R}$. Hence the stability analysis of the nonlinear parabolic evolution problem (1.1) reduces to the study of the aymptotic behaviour as $t \rightarrow \infty$ of the solutions of the Volterra integral equation (2.1). It turns out that the nonlocality of the problem is thereby encoded in the kernel $a$ in a more tractable way.

Proposition 2.3. For fixed $\beta \in(0, \infty)$ consider orbits $\Phi_{\beta}\left(\cdot, u_{0}\right)$ of the semiflow $\left(\Phi_{\beta}, \mathrm{H}^{1}\right)$. Then, for any $u_{0} \in \mathrm{H}^{1}$, it holds that

$$
\Phi_{\beta}\left(t, u_{0}\right) \longrightarrow 0 \text { in } \mathrm{H}^{1} \Longleftrightarrow \gamma_{\pi}\left(\Phi_{\beta}\left(t, u_{0}\right)\right) \longrightarrow 0 \text { in } \mathbb{R},
$$

as $t \rightarrow \infty$.

Proof. " $\Rightarrow$ ": If $\Phi_{\beta}\left(t, u_{0}\right) \rightarrow 0$ as $t \rightarrow \infty$, then the fact that $\gamma_{\pi} \in \mathcal{L}\left(\mathrm{H}^{1}, \mathbb{R}\right)$ implies the continuity of $\gamma_{\pi}$ and thus $\gamma_{\pi}\left(\Phi_{\beta}\left(t, u_{0}\right)\right) \rightarrow 0$ as $t \rightarrow \infty$.

" $\Leftarrow$ ": If $u(t, \pi) \rightarrow 0$ as $t \rightarrow \infty$, then by (1.3) and (2.1) we have that

$$
\lim _{t \rightarrow \infty}\left[u_{N}(t, \pi)+\int_{0}^{t} a(t-\tau) g_{\beta}(u(\tau, \pi)) d \tau\right]=0
$$

which entails the convergence of the integral addend since $u_{N}(t, \pi) \rightarrow \bar{u}_{0}$ as $t \rightarrow \infty$. We claim that the limit can be identified, i.e. that

$$
\lim _{t \rightarrow \infty} \int_{0}^{t} a(t-\tau) g_{\beta}(u(\tau, \pi)) d \tau=-\frac{1}{\pi} \int_{0}^{\infty} g_{\beta}(u(\tau, \pi)) d \tau .
$$

Indeed, by assumption, given any $\varepsilon>0$, a time $t_{\varepsilon}>0$ can be found such that

$$
\left|g_{\beta}(u(\tau, \pi))\right| \leq \varepsilon \text { for } \tau \geq t_{\varepsilon}
$$

since $g_{\beta}(0)=0$ and $g_{\beta}$ is continuous. Then, for any $t>t_{\varepsilon}$ we have that

$$
\int_{0}^{t} a(t-\tau) g_{\beta}(u(\tau, \pi)) d \tau=-\frac{1}{\pi} \int_{0}^{t} g_{\beta}(u(\tau, \pi)) d \tau+\int_{0}^{t}\left[a(t-\tau)+\frac{1}{\pi}\right] g_{\beta}(u(\tau, \pi)) d \tau .
$$

Next we use the fact that we know the full spectral resolution of the operator $A_{N}$ given by

$$
A_{N} u=\sum_{k=1}^{\infty} k^{2} \underbrace{\left\langle u, \varphi_{k}\right\rangle}_{\hat{u}_{k}} \varphi_{k}, u \in \operatorname{dom}\left(A_{N}\right)
$$

where the eigenfunctions are given by $\varphi_{k}(x)=\sqrt{\frac{2}{\pi}} \cos (k x)$ for $k \in \mathbb{N}$ and $x \in[0, \pi]$ and $\varphi_{0} \equiv \frac{1}{\sqrt{\pi}}$. This yields a representation of the semigroup as

$$
e^{-t A_{N}} u_{0}=\bar{u}_{0}+\sum_{k=1}^{\infty} \hat{u}_{0 k} e^{-t k^{2}} \varphi_{k} .
$$

Now observe that

$$
\delta_{0}=\frac{1}{\pi}+\sum_{k=1}^{\infty}\left\langle\delta_{0}, \varphi_{k}\right\rangle \varphi_{k}=\frac{1}{\pi}+\sqrt{\frac{2}{\pi}} \sum_{k=1}^{\infty} \varphi_{k}
$$

with convergence in $\mathrm{H}^{-1}$, which yields

$$
a(t)=-\left(e^{-t A_{N}} \delta_{0}\right)(\pi)=-\frac{1}{\pi}-\sqrt{\frac{2}{\pi}} \sum_{k=1}^{\infty} e^{-t k^{2}} \varphi_{k}(\pi)=-\frac{1}{\pi}+\frac{2}{\pi} \sum_{k=1}^{\infty}(-1)^{k+1} e^{-t k^{2}} .
$$


Next we split the integral

$$
\int_{0}^{t}\left[a(t-\tau)+\frac{1}{\pi}\right] g_{\beta}(u(\tau, \pi)) d \tau
$$

into the integral up to $t_{\varepsilon}$ and the rest. Estimating separately, we see that

$$
\begin{aligned}
\left|\int_{0}^{t_{\varepsilon}} g_{\beta}(u(\tau, \pi))\left[a(t-\tau)+\frac{1}{\pi}\right] d \tau\right| & \leq \frac{2}{\pi} \sum_{k=1}^{\infty} \int_{0}^{t_{\varepsilon}} e^{-(t-\tau) k^{2}} d \tau \\
& \leq \frac{2}{\pi} \sum_{k=1}^{\infty} \frac{1}{k^{2}} e^{-\left(t-t_{\varepsilon}\right) k^{2}} \leq c e^{-\left(t-t_{\varepsilon}\right)}, t>t_{\varepsilon}
\end{aligned}
$$

since $\left\|g_{\beta}\right\|_{\infty} \leq 1$, and that

$$
\begin{aligned}
\left|\int_{t_{\varepsilon}}^{t} g_{\beta}(u(\tau, \pi))\left[a(t-\tau)+\frac{1}{\pi}\right] d \tau\right| & \leq c \sum_{k=1}^{\infty} \int_{t_{\varepsilon}}^{t}\left|g_{\beta}(u(\tau, \pi))\right| e^{-(t-\tau) k^{2}} d \tau \\
& \leq c \varepsilon \sum_{k=1}^{\infty} \frac{1}{k^{2}}\left(1-e^{-\left(t-t_{\varepsilon}\right) k^{2}}\right) \leq c \varepsilon, t>t_{\varepsilon} .
\end{aligned}
$$

This allows us to conclude that, given $\varepsilon>0$, there is $\tilde{t}_{\varepsilon}>0$ such that

$$
\left|\int_{0}^{t} a(t-\tau) g_{\beta}(u(\tau, \pi)) d \tau+\frac{1}{\pi} \int_{0}^{t} g_{\beta}(u(\tau, \pi)) d \tau\right| \leq \varepsilon
$$

for $t>\tilde{t}_{\varepsilon}$, which yields the stated convergence. Next notice that, given $x \in[0, \pi)$, it holds that

$$
u(t, x)=u_{N}(t, x)+\int_{0}^{t} a(t-\tau, x) g_{\beta}(u(\tau, x)) d \tau,
$$

where

$$
a(t, x)=-\left(e^{-t A_{N}} \delta_{0}\right)(x)=-\frac{1}{\pi}+\frac{2}{\pi} \sum_{k=1}^{\infty} \cos (k x) e^{-t k^{2}} \text { for } t \geq 0 .
$$

A similar argument then shows that also for $x \in[0, \pi)$ and $t \rightarrow \infty$

$$
u(t, x) \rightarrow \bar{u}_{0}-\frac{1}{\pi} \int_{0}^{\infty} g_{\beta}(u(\tau, \pi)) d \tau=0 .
$$

The limit is 0 since we already proved that

$$
\bar{u}_{0}=\frac{1}{\pi} \int_{0}^{\infty} g_{\beta}(u(\tau, \pi)) d \tau
$$

in the first step of the proof. Note that, while $a(t, 0)$ has a singularity in $t=0$, this singularity is integrable, as shown in Remark 2.2(c), and the argument goes through. We have thus shown that $\Phi_{\beta}\left(t, u_{0}\right)(x) \longrightarrow 0$ as $t \rightarrow \infty$ pointwise for each $x \in[0, \pi]$. We now prove that convergence takes place in the topology of $\mathrm{H}^{1}$. To that end use (1.3) to derive the equation satisfied by $\hat{u}_{n}(t)$, which is the $n$-th coefficient in the expansion of the solution

$$
u(t, x)=\sum_{k=1}^{\infty}\left\langle u(t, \cdot), \varphi_{k}\right\rangle \varphi_{k}(x)=\sum_{k=1}^{\infty} \hat{u}_{k}(t) \varphi_{k}(x),
$$

Observe that the $\mathrm{H}^{1}$ norm of a function $u$ as above is equivalent to

$$
\|u\|_{\mathrm{H}^{1}}^{2}=\sum_{k=0}^{\infty}\left(1+k^{2}\right)\left|\hat{u}_{k}\right|^{2} .
$$

This is seen by extending $u$ to a periodic function $\tilde{u}$ by reflection, i.e. by setting $\tilde{u}(x)=u(2 \pi-x)$ for $x \in[\pi, 2 \pi]$, and noticing the direct relation between the standard Fourier series of $\tilde{u}$ and the spectral 
basis expansion of $u$. We also use the fact that $u \in \mathrm{H}^{1}(0, \pi)$ if and only if $\tilde{u} \in \mathrm{H}_{p e r}^{1}(0,2 \pi)$. For $n=0$ we have that

$$
\hat{u}_{0}(t)=\bar{u}(t)=\bar{u}_{0}-\frac{1}{\pi} \int_{0}^{t} g_{\beta}(u(\tau, \pi)) d \tau
$$

which converges to zero as $t$ approaches infinity as we have seen above. For $n \geq 1$ one has that

$$
\hat{u}_{n}(t)=e^{-t n^{2}} \hat{u}_{0 n}-\sqrt{\frac{2}{\pi}} \int_{0}^{t} g_{\beta}(u(\tau, \pi)) e^{-(t-\tau) n^{2}} d \tau .
$$

A simple calculation using the boundedness of $g_{\beta}$ then yields

$$
\left(1+n^{2}\right)\left|\hat{u}_{n}(t)\right|^{2} \leq c\left(1+n^{2}\right)\left|\hat{u}_{0 n}\right|+\frac{c}{n^{4}}\left(1+n^{2}\right), n \geq 1 .
$$

This, together with the fact that $u_{0} \in \mathrm{H}^{1}$, implies that the series

$$
\sum_{n \geq 1}\left(1+n^{2}\right)\left|\hat{u}_{n}(t)\right|^{2}
$$

converges uniformly in $t \geq 0$. Arguing as in the first part of the proof, i.e. by using the integral representation (1.3) and splitting it, we obtain

$$
\left(1+n^{2}\right)\left|\hat{u}_{n}(t)\right|^{2} \rightarrow 0 \text { as } t \rightarrow \infty,
$$

for any $n \geq 0$ and we can infer that $u(t) \rightarrow 0$ in $\mathrm{H}^{1}$ by combining this with the uniform convergence of the series (2.12). Note that the tail of the series can be made small uniformly in time, while the remaining finite sum can be estimated by the choice of a sufficiently large time.

\section{Relationship to Feedback Control Problems}

No reference will be made to this section in the rigorous development of the proof for the stability result. Nevertheless we include it before proceeding to the analysis of the asymptotic behaviour of the Volterra integral equation derived in the previous section. Our concern is that, otherwise, the proof may appear as a rather arbitrary succession of technical results for a Volterra integral equation, where, in the end, somewhat miraculously, the desired result emerges out of the blue. In addition the linear and streamlined exposition that results when omitting this section would not at all reflect the rather convoluted path which eventually led to this proof. Readers familiar with feedback control systems and distributed parameter systems, in particular those with knowledge of the celebrated Nyquist and Popov criteria may omit this section without fearing any gaps in rigour for the sequel of this study. We refer to [16], 4] for more information on feedback control systems.

A classical $n \in \mathbb{N}$-dimensional single-input-single-output (SISO) feedback system, which is also a special case of Lur'e system (which allows for multiple inputs and outputs), takes the form

$$
\left\{\begin{array}{l}
\dot{x}=A x+b u \\
y=c^{\top} x \\
u=h(y)
\end{array}\right.
$$

for a matrix $A \in \mathbb{R}^{n \times n}$, for vectors $b, c \in \mathbb{R}^{n \times 1}$, and for a nonlinear function $h: \mathbb{R} \rightarrow \mathbb{R}$. An important object in the study of such systems is the so-called transfer function $G$ given by

$$
G(s)=(s-A)^{-1} \mathcal{L}(h \circ y) .
$$

It is obtained from the system by taking a Laplace transform $\mathcal{L}$ with zero initial condition. Assuming $h(0)=0$ yields the equilibrium $y \equiv 0$ and one is interested in its stability. This has attracted a great deal of interest, especially for the class of nonlinearities satisfying a so-called local [global] sector condition, i.e. such that

$$
\alpha z^{2} \leq z h(z) \leq \beta z^{2} \text { for } z \in(a, b)[z \in \mathbb{R}]
$$


for real numbers $a<b$. Two stability conditions which emerged from the research go by the name of the circle and the Popov criteria, respectively. They can be formulated in terms of the transfer function of the system. The present paper has drawn inspiration from these developments of control theory in view of the possibility of thinking of $(1.2)$ as an infinite dimensional SISO feedback system. The correspondence is given by

$$
x \rightarrow u, A \rightarrow-A_{N}, b \rightarrow \delta_{0}, c^{\top} \rightarrow \gamma_{\pi}\left(=\delta_{\pi}^{\top}\right), \text { and } h(y) \rightarrow \tanh (\beta y),
$$

where $h$ satisfies a global sector condition with $\alpha=0$ and $\beta>0$.

The above control system is a closed loop feedback system. In feedback control a successful strategy consists in deducing the properties of a closed loop system from the analysis of the corresponding open loop one obtained by simply cutting the feedback loop. The obtained open loop system is then just an input-ouput black box without feedback. When the output of the black box depends linearly on the applied input a so-called open loop frequency scan across all pure sinusoidal input frequencies determines the amplitude and phase response of the output of the open loop system. The 2D open loop frequency response diagram where output amplitude and output phase are plotted across all frequencies is a workhorse in electrical and mechanical engineering. The method is known as the Bode or Nyquist stability plot. The importance of the Nyquist plot lies in the fact that the maximal feedback amplification parameter still preserving asymptotic stability of the rest state, the so-called maximal gain, can be determined from the transfer function of the open loop system. Often it is simply read off from a Nyquist plot determined by empirical frequency response measurements. The Popov criterion applies to a wide class of nonlinear feedback systems. It combines the frequency response of the linear open loop system via its transfer function with the sectorial parameters of the nonlinear feedback function. One single combined criterion formulated in terms of the location of a certain curve in the complex plane (the Popov plot) provides a flexible and often optimal stability analysis tool.

3.1. The nonlocal linear feedback problem. To illustrate this approach we first study the following linear open loop system related to problem (1.1)

$$
\begin{cases}u_{t}-u_{x x}=0 & \text { in }(0, \infty) \times(0, \pi), \\ u_{x}(t, 0)=-f(t) & \text { for } t \in(0, \infty), \\ u_{x}(t, \pi)=0 & \text { for } t \in(0, \infty) \\ u(0, \cdot) \equiv 0 & \text { in }(0, \pi)\end{cases}
$$

Here $f(t) \in \mathrm{BC}\left(\mathbb{R}^{+}, \mathbb{R}\right)$ is interpreted as the input to the system at $x=0$. Note that we choose the equilibrium state $u_{0} \equiv 0$ as the initial condition. The output is obtained by measuring the temperature at $x=\pi$ by taking the trace of the solution $u\left(t, u_{0}\right)$ of the initial value (3.1) which is given by

$$
u\left(t, u_{0}\right)=e^{-t A_{N}} u_{0}+\int_{0}^{t} e^{-(t-\tau) A_{N}} \gamma_{0}^{\prime}(f(\tau)) d \tau=\int_{0}^{t} f(\tau) e^{-(t-\tau) A_{N}} \delta_{0} d \tau
$$

Hence the system output $g(t)$ is given by

$$
g(t):=\gamma_{\pi}\left(u\left(t, u_{0}\right)\right)=\int_{0}^{t} f(\tau)\left[e^{-(t-\tau) A_{N}} \delta_{0}\right](\pi) d \tau
$$

In the previous section we discussed that

$$
a(t)=-\left[e^{-t A_{N}} \delta_{0}\right](\pi)=-\frac{1}{\pi}+\frac{2}{\pi} \sum_{k=1}^{\infty}(-1)^{k+1} e^{-t k^{2}}
$$

and therefore the output is computed by convolution of the input with the kernel $-a$

$$
g(t)=-\int_{0}^{t} a(t-\tau) f(\tau) d \tau=(-a * f)(t)
$$


We can now apply the Laplace transform to both sides of (3.11) to obtain

$$
\mathcal{L}(g)=\mathcal{L}(-a) \mathcal{L}(f)
$$

The transfer function is defined as $G_{\text {nloc }}:=\mathcal{L}(-a)$ and was already computed in the previous section

$$
G_{\text {nloc }}(s)=\mathcal{L}\left(\frac{1}{\pi}+\frac{2}{\pi} \sum_{k=1}^{\infty}(-1)^{k} e^{-t k^{2}}\right)(s)=\frac{1}{\pi s}+\frac{2}{\pi} \sum_{k=1}^{\infty} \frac{(-1)^{k}}{s+k^{2}}=\frac{1}{\sqrt{s} \sinh (\pi \sqrt{s})} .
$$

The closed loop feedback system with gain $\beta$ corresponding to 3.1 is given by

$$
\begin{cases}u_{t}-u_{x x}=0 & \text { in }(0, \infty) \times(0, \pi), \\ u_{x}(t, 0)=\beta u(t, \pi) & \text { for } t \in(0, \infty), \\ u_{x}(t, \pi)=0 & \text { for } t \in(0, \infty), \\ u(0, \cdot) \equiv 0 & \text { in }(0, \pi),\end{cases}
$$

In feedback control analysis, the closed loop system transfer function is obtained from the open loop transfer function. Adopting the usual definition from finite dimensional feeback systems heuristically, we set

$$
G_{\text {nloc }}^{\text {cl-loo }}(\cdot, \beta)=\frac{G_{\text {nloc }}}{1+\beta G_{\text {nloc }}} .
$$

It is clear that the zeros of the closed loop transfer function and the zeros of the open loop transfer function coincide. The poles of the closed loop transfer function correspond to the set

$$
\left\{s \in \mathbb{C} \mid \beta G_{\text {nloc }}(s)=-1\right\} \text {. }
$$

This leads to the use of Rouché's theorem to derive the Nyquist stability criterion. It gives sufficient conditions for the stability of the trivial equilibrium in terms of a vanishing winding number of the Nyquist curve

$$
\operatorname{Nyquist}\left(\beta G_{\text {nloc }}\right):=\left\{\beta G_{\text {nloc }}(i \omega) \mid \omega \in \mathbb{R}\right\},
$$

around the point $(-1,0)$ in the complex plane. In Figure 1 the Nyquist plot is computed numerically for $\beta=1$ from the representation

$$
G_{\mathrm{nloc}}(i \omega)=\frac{1}{\sqrt{i \omega} \sinh (\pi \sqrt{i \omega})}
$$

for numerical values in $\omega \in(-50,-0.2) \cup(0.2,50)$. A semi-circle in the complex right half-plane of radius 0.2 is followed to avoid the singularity at zero. This leads to a closed Nyquist curve. A solution $\left(\omega_{0}, \beta_{0}\right)$ such that $G_{\text {nloc }}\left(i \omega_{0}\right) \in(-\infty, 0)$ and

$$
\beta_{0} G_{\text {nloc }}\left(i \omega_{0}\right)=-1
$$

leads to a critical parameter value $\beta_{0}$. It can be found from the condition

$$
\operatorname{Im}\left[G_{\text {nloc }}\left(i \omega_{0}\right)\right]=-\frac{1}{\pi \omega_{0}}-\frac{2}{\pi} \sum_{k=1}^{\infty}(-1)^{k} \frac{\omega_{0}}{\omega_{0}^{2}+k^{4}}=0
$$

or, equivalently, from

$$
\sum_{k=1}^{\infty} \frac{(-1)^{k-1}}{1+k^{4} / \omega_{0}^{2}}=\frac{1}{2}
$$

It appears that (3.8) possesses infintely many real solutions. In fact, by using the explicit representation (3.7) a calculation that separates real and imaginary parts of the denumerator in (3.7) and uses the expression for the imaginary part of $\frac{1}{a+i b}$ leads to an explicit equivalent expression for the condition 3.8 , given by

$$
\sinh \left(\pi \sqrt{\frac{\omega}{2}}\right) \cos \left(\pi \sqrt{\frac{\omega}{2}}\right)+\cosh \left(\pi \sqrt{\frac{\omega}{2}}\right) \sin \left(\pi \sqrt{\frac{\omega}{2}}\right)=0
$$




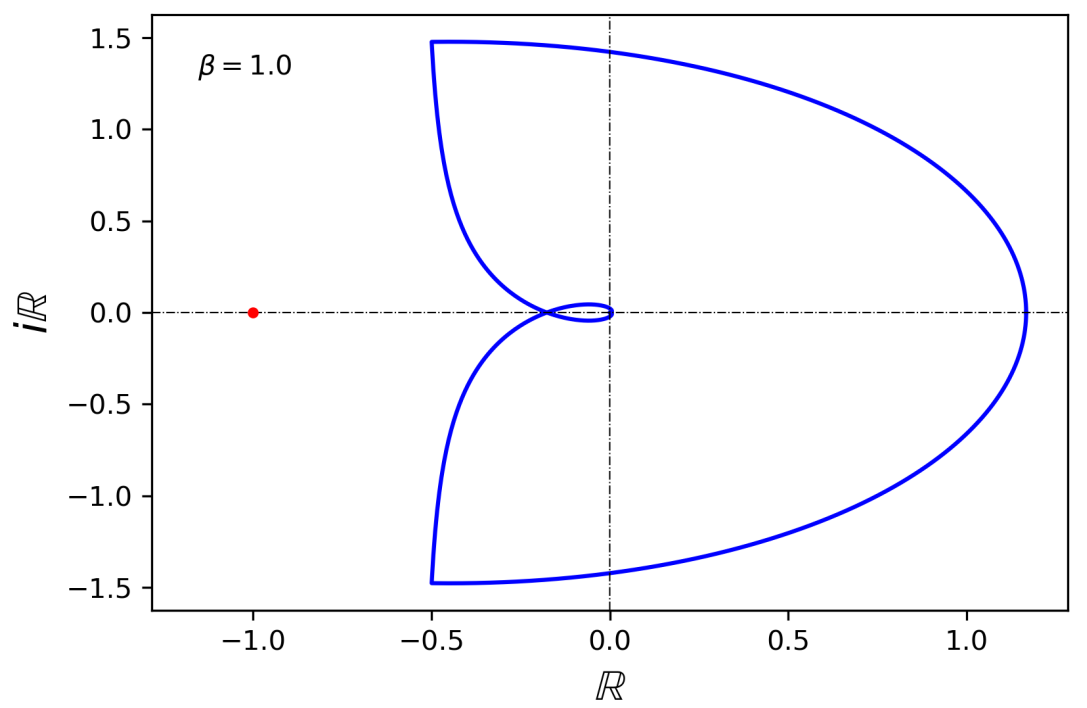

Figure 1. Nyquist plot Nyquist $\left(G_{\text {nloc }}\right)$. The limited resolution hides the infinitely many windings of the curve around the origin as $\omega \rightarrow \pm \infty$.

Clearly this is equivalent to the condition already found in [1] (Remarks 4.2.)

$$
\tan \left(\pi \sqrt{\frac{\omega}{2}}\right)=-\tanh \left(\pi \sqrt{\frac{\omega}{2}}\right)
$$

and shows that (3.8) has infinitely many solutions.

Picking the smallest solution, which occurs for $\omega_{0} \approx 1.13344388$, it is found by inserting, that $G_{\text {nloc }}\left(i \omega_{0}\right) \approx-0.17650842$. The critical value for $\beta_{0}$ is finally obtained from

$$
\beta_{0}=-\frac{1}{G_{\mathrm{nloc}}\left(i \omega_{0}\right)}=-\sqrt{i \omega_{0}} \sinh \left(\pi \sqrt{i \omega_{0}}\right) \approx 5.6655
$$

Hence this formal application of the Nyquist criterion suggests that the zero state of $(3.6)$ is asymptotically stable for $\beta \in\left(0, \beta_{0}\right)$. This stability interval determined heuristically is identical to the (linear) stability interval determined rigorously in [11] by fully describing the spectrum of the generator of the linear semigroup associated with (3.6).

3.2. The local linear feedback problem. We also briefly discuss the local version of (3.1) and also refer to [4] where a similar stability analysis of this problem can be found. We start again with an input signal $f(t)$ that enters as before:

$$
\begin{cases}u_{t}-u_{x x}=0 & \text { in }(0, \infty) \times(0, \pi), \\ u_{x}(t, 0)=-f(t) & \text { for } t \in(0, \infty), \\ u_{x}(t, \pi)=0 & \text { for } t \in(0, \infty) \\ u(0, \cdot) \equiv 0 & \text { in }(0, \pi)\end{cases}
$$

Now the output is obtained by measuring the temperature at $x=0$ by taking the trace $\gamma_{0}$ of the solution $u\left(t, u_{0}\right)$ of the initial value $(3.9)$. Hence the system output $g(t)$ is given by

$$
g(t):=\gamma_{0}\left(u\left(t, u_{0}\right)\right)=\int_{0}^{t} f(\tau)\left[e^{-(t-\tau) A_{N}} \delta_{0}\right](x=0) d \tau .
$$




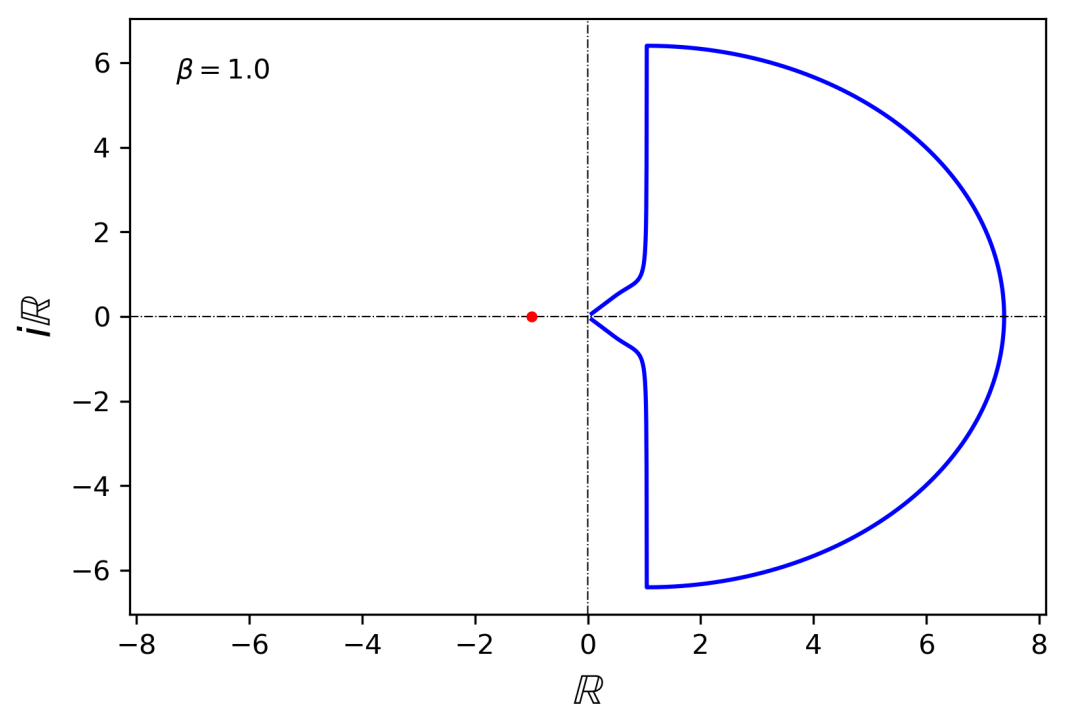

Figure 2. Nyquist plot of the transfer function $G_{\text {loc }}$.

For this local version we now determine the kernel by evaluating at $x=0$

$$
a_{\mathrm{loc}}(t):=-\left[e^{-t A_{N}} \delta_{0}\right](x=0)=-\frac{1}{\pi}-\frac{2}{\pi} \sum_{k=1}^{\infty} e^{-t k^{2}} .
$$

Again the output is given by convoluting the input with the kernel $a_{\text {loc }}$

$$
g(t)=-\int_{0}^{t} a_{\mathrm{loc}}(t-\tau) f(\tau) d \tau=\left(-a_{\mathrm{loc}} * f\right)(t)
$$

and by applying the Laplace transform

$$
\mathcal{L}(g)=\mathcal{L}\left(-a_{\text {loc }}\right) \mathcal{L}(f)
$$

The transfer function is defined as $G_{\text {loc }}:=\mathcal{L}\left(-a_{\text {loc }}\right)$ and it can be expressed as

$$
G_{\text {loc }}(s)=\mathcal{L}\left(\frac{1}{\pi}+\frac{2}{\pi} \sum_{k=1}^{\infty} e^{-t k^{2}}\right)(s)=\frac{1}{\pi s}+\frac{2}{\pi} \sum_{k=1}^{\infty} \frac{1}{s+k^{2}}=\frac{\cosh (\pi \sqrt{s}))}{\sqrt{s} \sinh (\pi \sqrt{s})} .
$$

The local closed loop feedback system with gain $\beta$ is now given by

$$
\begin{cases}u_{t}-u_{x x}=0 & \text { in }(0, \infty) \times(0, \pi), \\ u_{x}(t, 0)=\beta u(t, 0) & \text { for } t \in(0, \infty), \\ u_{x}(t, \pi)=0 & \text { for } t \in(0, \infty), \\ u(0, \cdot) \equiv 0 & \text { in }(0, \pi) .\end{cases}
$$

In Figure 2 we compute the Nyquist plot from the representation (3.13) and observe that the Nyquist plot lies in the right complex half-plane. Therefore the stability range for the local problem obtained from the above heuristic application of the Nyquist criterion is $\beta \in(0, \infty)$. We point out that this already follows rigorously from the analysis of the spectrum of the generator of the linear semigroup, as is discussed in [11] and [12. In fact, for a wide class of nonlinear parabolic evolution problems in one space dimension with "local" or "separated" boundary conditions, the general results of P. Poláčik et. al. (see e.g. [3]) show that all orbits converge to steady states, whenever a compact global attractor is known to exist. 
3.3. Nyquist criterion and spectral linear stability analysis. This short section aims at drawing a formal connection (with no proofs) between the mathematical linear stability analysis originally performed in [11] and the Nyquist diagram approach found in applications and just described. It is known from semigroup theory [9] that the Laplace transform of a strongly continuous semigroup $\left\{T_{A}(t) \mid t \geq 0\right\}$ on a Banach space $E$ is the resolvent of its generator, i.e. that

$$
\mathcal{L}\left(T_{A}(\cdot)\right)(s)=(s-A)^{-1} \text { for } s \in \rho(A),
$$

with $s \geq \omega(A)$, where $\omega(A)$ is the smallest real number for which $\left\|T_{A}(t)\right\|_{\mathcal{L}(E)} \leq M e^{\omega t}$ for $t \geq 0$ and some constant $M \geq 1$. This formula hints at the fact that the poles of the resolvent are the modes of decay/growth of $T_{A}$, which, in turn, correspond to the eigenvalues of $A$. For the semigroup $e^{-\cdot A_{N}}$ the eigenvalues $\lambda \in \mathbb{C}$ are determined by the equation $\sqrt{\lambda} \sin (\pi \sqrt{\lambda})=\beta$ as follows from [11], while $\bar{u}=\mathcal{L}(u)$ satisfies the identity

$$
\bar{u}(s, x)=\left[\left(s+A_{N}\right)^{-1} u_{0}\right](x)-\beta \bar{u}(s, \pi)\left[\left(s+A_{N}\right)^{-1} \delta_{0}\right](x)
$$

as follows from the weak formulation

$$
\left(s+A_{N}\right) \bar{u}=u_{0}-\beta \bar{u}(\pi) \delta_{0} \text { in } \mathrm{H}^{-1}, \bar{u} \in \mathrm{H}^{1},
$$

of the Laplace transform of (1.1) given by

$$
\left\{\begin{array}{l}
s \bar{u}-u_{0}-\bar{u}_{x x}=0 \quad \text { in }(0, \pi) \\
\bar{u}_{x}(0)=\beta \bar{u}(\pi) \\
\bar{u}_{x}(\pi)=0
\end{array}\right.
$$

which confirms that

$$
\mathcal{L}\left(e^{-t A_{N}} \delta_{0}\right)(s)=\left[\left(s+A_{N}\right)^{-1} \delta_{0}\right](\pi)=\frac{1}{\sqrt{s} \sinh (\pi \sqrt{s})}
$$

is the transfer function of the open loop control system. Equation (3.15) allows one to recover $\bar{u}(s, \pi)$ by simple evaluation at $x=\pi$ to get

$$
\bar{u}(s, \cdot)=\left(s+A_{N}\right)^{-1} u_{0}-\beta \frac{\left[\left(s+A_{N}\right)^{-1} u_{0}\right](\pi)}{1+\beta\left[\left(s+A_{N}\right)^{-1} \delta_{0}\right](\pi)}\left(s+A_{N}\right)^{-1} \delta_{0},
$$

an explicit representation of the solution of the closed loop system. Notice that, after evaluation at $x=\pi$, equation (3.15) is simply the Laplace transform of the Volterra integral equation (2.1) in the linear case. Now the modes of decay (or growth) of $u$ are fully determined by the poles of $\bar{u}$, which, in turn, are determined by the zeros of $1+\beta\left[\left(s+A_{N}\right)^{-1} \delta_{0}\right](\pi)$, since these are the only poles of $\bar{u}$ which change with $\beta$. Since the Nyquist plot is a complex analytical procedure to identify poles in the "bad" half-plane, it ends up being a method to check when stability is lost for the linear problem and, hence, amounts to a linearized stability criterion for the original nonlinear system, in this case.

3.4. The Popov criterion applied to the nonlocal and nonlinear feedback problem. The Popov criterion for a nonlinearity satisfying the global sector condition $[0, \beta]$ is stated in terms of the transfer function of the linear open loop system. Applied to our transfer function the Popov criterion requires that, for a given parameter $\beta>0$, there exists a constant $q(\beta)>0$ such that, for $\omega \in \mathbb{R}$,

$$
\operatorname{Re}\left[G_{\text {nloc }}(i \omega)\right]-q \omega \operatorname{Im}\left[G_{\text {nloc }}(i \omega)\right]>-\frac{1}{\beta} .
$$

If we define the Popov plot by

$$
P\left(G_{\text {nloc }}\right):=\left\{\operatorname{Re}\left[G_{\text {nloc }}(i \omega)\right]+i \omega \operatorname{Im}\left[G_{\text {nloc }}(i \omega)\right] \mid \omega>0\right\}
$$

then (3.16) is equivalent to the existence of a constant $q>0$ such that the straight line in the complex plane with slope $q$ through the real point $-\frac{1}{\beta}$ lies to the left of the Popov plot $P\left(G_{\text {nloc }}\right)$. If the criterion is fulfilled, then any nonlinearity in the sector $[0, \beta]$ leads to an asymptotically stable 
nonlinear feedback system. Note that our particular nonlinearity $\tanh (\beta \cdot)$ satifies the sector condition $[0, \beta]$ and therefore the nonlinear evolution problem $(1.1)$, at least formally, falls into the class of nonlinear feedback systems covered by the Popov criterion.

The Popov plot (3.17) can easily be computed numerically from the explicit representation (3.13) and is displayed in Figure 3 . Since the Popov plot crosses the real line at $-\frac{1}{\beta_{0}}$ where $\beta_{0} \approx 5.6655$ is the constant already discussed above, the criterion suggests that the parameter range of stability is given by $\left(0, \beta_{0}\right)$. In the next section we will prove this result by studying the nonlinear Volterra integral equation (2.1).

In the course of our rigorous analysis, which will be resumed in the next section, the application of the Parseval-Plancherel identity will lead to a Fourier representation of a stability condition in terms of the kernel of the integral equation. The stability condition obtained in this way then turns out to be equivalent to the Popov criterion and allows for a rigorous proof of the above heuristic argument via the integral equation.

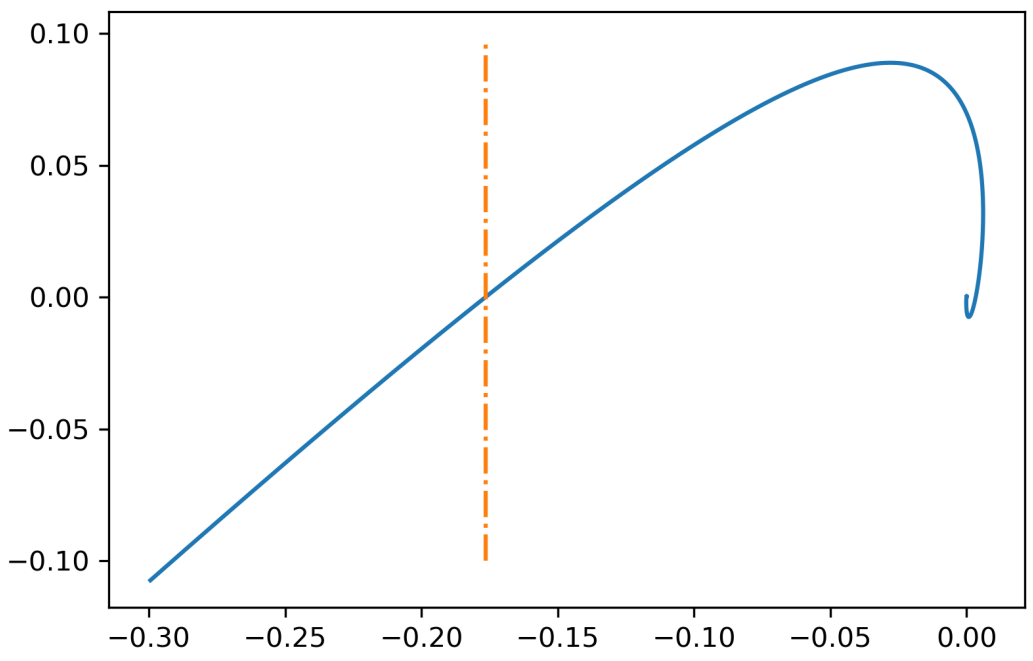

Figure 3. Popov plot $P\left(G_{\text {nloc }}\right)$. The limited resolution hides the infinitely many windings of the curve around the origin as $\omega \rightarrow \infty$.

\section{Asymptotic Stability for the Integral Equation}

The objective of this section is the proof of the following result. The ideas originate in [20] and are adapted to obtain the following proposition. In particular, we have adapted the expression for $W_{1}$ in [20] by subtracting the $\beta$-dependent term.

Proposition 4.1. Fix $\beta \in\left(0, \beta_{0}\right)$ and $u_{0} \in \mathrm{H}^{1}$. Let $y \in \mathrm{BC}((0, \infty), \mathbb{R})$ be a solution of the integral equation 2.1. Then $\lim _{t \rightarrow \infty} y(t)=0$.

For the proof we need a series of lemmas and an auxiliary function.

Definition 4.2. For $\beta, q \in(0, \infty)$ and $y \in \mathrm{BC}([0, \infty), \mathbb{R})$ set

$$
W_{\beta, q}(y)(t):=\sum_{i=1}^{3} W_{i}(y)(t), t \geq 0,
$$


where

$$
\begin{aligned}
& W_{1}(y)(t):=\int_{0}^{t} g_{\beta}(y(\tau))\left[y(\tau)-\frac{g_{\beta}(y(\tau))}{\beta}\right] d \tau, \\
& W_{2}(y)(t):=q G_{\beta}(y(t)) \text { for } G_{\beta}(z):=\int_{0}^{z} g_{\beta}(\zeta) d \zeta, \\
& W_{3}(y)(t):=\frac{1}{2 \pi}\left(\int_{0}^{t} g_{\beta}(y(\tau)) d \tau\right)^{2} .
\end{aligned}
$$

Note that in our notation we may not always explicitly indicate the dependence on $\beta$ and $q$ in the notation for $W_{i}, i=1,2,3$ nor the dependence on the function $y$.

Lemma 4.3. Let $\beta, q \in(0, \infty)$ and $y \in \mathrm{BC}([0, \infty), \mathbb{R})$. Then

$$
W_{i}(t) \geq 0, t \geq 0, i=1,2,3 .
$$

It therefore also holds that $W_{\beta, q} \geq 0$ for $t \geq 0$.

Proof. The nonnegativity of $W_{1}$ follows from its nonnegative integrand. First note that $g_{\beta}(y)=0$ for $y=0$ and hence the integrand vanishes for $y=0$. For $y \neq 0$ we can express the integrand as

$$
\frac{1}{\beta} g_{\beta}(y) \beta y\left[1-\frac{g_{\beta}(y)}{\beta y}\right]
$$

and the positivity of this expression follows from $g_{\beta}(y) \beta y>0$ and from $\frac{g_{\beta}(y)}{\beta y} \in(0,1)$. Also the nonnegativity of $W_{2}$ is a consequence of $g_{\beta}(y) y>0$ for $y \neq 0$.

Lemma 4.4. Let $\beta, q \in(0, \infty)$ and let $y \in \mathrm{BC}((0, \infty), \mathbb{R})$ be a solution of the integral equation (2.1) with $u_{0} \in \mathrm{H}^{1}$. Then

$$
W_{\beta, q}(t)=V_{\beta, q}(t)+R_{\beta, q}(t), t \geq 0,
$$

where

$$
V_{\beta, q}(t):=\int_{0}^{t} g_{\beta}(y(\tau))\left[f(\tau)+q f^{\prime}(\tau)\right] d \tau+q G_{\beta}(y(0))
$$

and

$$
R_{\beta, q}(t):=\int_{0}^{t} g_{\beta}(y(\tau))\left\{\int_{0}^{\tau}\left[a_{s}(\tau-\sigma)+q a_{s}^{\prime}(\tau-\sigma)\right] g_{\beta}(y(\sigma)) d \sigma-\frac{g_{\beta}(y(\tau))}{\beta}\right\} d \tau .
$$

Using convolutions, the latter expression can be written more concisely as

$$
R_{\beta, q}(t)=\int_{0}^{t} g_{\beta}(y(\tau)) J_{\beta, q}(\tau) d \tau
$$

where $J_{\beta, q}$ is defined for $\tau \geq 0$ by

$$
J_{\beta, q}:=\left[a_{s}+q a_{s}^{\prime}\right] * g_{\beta}(y(\cdot))-\frac{g_{\beta}(y(\cdot))}{\beta} .
$$

Proof. First note that by (2.1) and since $a(0)=0$ by Remark 2.2 we obtain

$$
y^{\prime}(t)=f^{\prime}(t)+a(0) g_{\beta}(y(t))+\int_{0}^{t} a^{\prime}(t-\tau) g_{\beta}(y(\tau)) d \tau=f^{\prime}(t)+\int_{0}^{t} a^{\prime}(t-\tau) g_{\beta}(y(\tau)) d \tau .
$$

Then by the simple transformation $d y=y^{\prime}(\tau) d \tau$ we can rewrite $W_{2}$ as

$$
G_{\beta}(y(t))=G_{\beta}(y(0))+\int_{0}^{t} g_{\beta}(y(\tau)) y^{\prime}(\tau) d \tau .
$$

By partial integration we can rewrite $W_{3}$ as

$$
W_{3}(y)(t)=\frac{1}{\pi} \int_{0}^{t}\left[\int_{0}^{\tau} g_{\beta}(y(\sigma)) d \sigma\right] g_{\beta}(y(\tau)) d \tau .
$$


Now insert (4.3) in (4.4) and use the above expressions $(4.3)-(4.5)$ in the definition of W. The claimed decomposition is obtained by collecting the integrals contaning $f$ and $f^{\prime}$ and the constant $q G_{\beta}(0)$, which produces $V_{\beta, q}$. The remainining terms are found to be equal to $R_{\beta, q}$ by using the identity $a_{s}=a+\frac{1}{\pi}$ to write

$$
\int_{0}^{\tau} a(\tau-\sigma) g_{\beta}(y(\sigma)) d \sigma+\frac{1}{\pi} \int_{0}^{\tau} g_{\beta}(y(\sigma)) d \sigma=\int_{0}^{\tau} a_{s}(\tau-\sigma) g_{\beta}(y(\sigma)) d \sigma .
$$

and by replacing $a^{\prime}$ by $a_{s}^{\prime}$ in the other term so that finally the kernel $a_{s}+q a_{s}^{\prime}$ results in the expression for $R_{\beta, q}$.

The more concise representation of $R_{\beta, q}$ is clear, since we simply use the definition of the convolution in our specific situation

$$
\left(\left[a_{s}+q a_{s}^{\prime}\right] * g_{\beta}(y(\cdot))\right)(\tau)=\int_{0}^{\tau}\left[a_{s}(\tau-\sigma)+q a_{s}^{\prime}(\tau-\sigma)\right] g_{\beta}(y(\sigma)) d \sigma .
$$

In the next lemma we apply the Parseval-Plancherel Theorem to derive a Fourier representation for $R_{\beta, q}$. This will allow us to obtain the criterion that makes $R_{\beta, q}<0$ so that the nonnegative quantity $W_{\beta, q}$ will be bounded by $V_{\beta, q}$ from above.

Lemma 4.5. Let $\beta, q \in(0, \infty)$ and let $y \in \mathrm{BC}((0, \infty), \mathbb{R})$ be a solution of the integral equation (2.1) with $u_{0} \in \mathrm{H}^{1}$. Then

$$
R_{\beta, q}(t)=\frac{1}{2 \pi} \int_{-\infty}^{\infty} \widehat{g}_{\beta, \theta_{t}}^{2}(\omega)\left[\hat{a}_{s}(\omega)+q \widehat{a_{s}^{\prime}}(\omega)-\frac{1}{\beta}\right] d \omega, t \geq 0,
$$

where for $\tau \in \mathbb{R}$ and $t \geq 0$

$$
g_{\beta, \theta_{t}}(\tau):=g_{\beta}(y(\tau)) \theta_{t}(\tau)
$$

with

$$
\theta_{t}(\tau):= \begin{cases}1, & \tau \in[0, t] \\ 0, & \tau \in \mathbb{R} \backslash[0, t]\end{cases}
$$

and $y(\tau):=0$ for $\tau<0$.

The Fourier transforms of $a_{s}$ and $a_{s}^{\prime}$ were discussed in Remarks 2.2 . Since $g_{\beta, \theta_{t}} \in \mathrm{L}^{1}(\mathbb{R}) \cap \mathrm{L}^{2}(\mathbb{R})$ for each $t \geq 0$, its Fourier transform is defined classically.

Proof. We will use the Parseval-Plancherel identity

$$
(f, g)_{2}=\int_{\mathbb{R}} f(x) g(x) d x=\frac{1}{2 \pi} \int_{\mathbb{R}} \hat{f}(\omega) \hat{g}(\omega) d \omega=\frac{1}{2 \pi}(\hat{f}, \hat{g})_{2},
$$

valid for $f, g \in \mathrm{L}^{2}(\mathbb{R})$. By setting

$$
J_{\beta, q}^{t}:=\left[a_{s}+q a_{s}^{\prime}\right] * g_{\beta, \theta_{t}}-\frac{g_{\beta, \theta_{t}}}{\beta} .
$$

we extend $J_{\beta, q}$ defined in 4.2 on $[0, \infty)$ to $\mathbb{R}$. Then $J_{\beta, q}^{t} \in \mathrm{L}^{1}(\mathbb{R}) \cap \mathrm{L}^{2}(\mathbb{R})$ for each $t \geq 0$ and the Parseval-Plancherel identity yields

$$
R_{\beta, q}(t)=\left(g_{\beta, \theta_{t}}, J_{\beta, q}^{t}\right)_{2}=\frac{1}{2 \pi}\left(\widehat{g_{\beta, \theta_{t}}}, \widehat{J_{\beta, q}^{t}}\right)_{2}=\frac{1}{2 \pi}\left({\widehat{g_{\beta, \theta_{t}}}}^{2}, \hat{a}_{s}+q{\widehat{a_{s}^{\prime}}}^{-\frac{1}{\beta}}\right)_{2} .
$$


Remark 4.6. For $\beta, q \in(0, \infty)$ and a solution $y \in \mathrm{BC}((0, \infty), \mathbb{R})$ of the integral equation (2.1) with $u_{0} \in \mathrm{H}^{1}$, the condition

$$
R_{\beta, q}(t)<0 \text { for } t \geq 0
$$

is satisfied if

$$
\hat{a}_{s}(\omega)+q \widehat{a_{s}^{\prime}}(\omega)-\frac{1}{\beta}<0 \text { for } \omega \in \mathbb{R}
$$

By using the series representation (2.3) one sees that this is equivalent to (2.10) and amounts to

$$
\frac{2}{\pi} \sum_{k=1}^{\infty}(-1)^{k+1}\left[\frac{k^{2}+q \omega^{2}}{k^{4}+\omega^{2}}\right]-\frac{q}{\pi}-\frac{1}{\beta}<0
$$

for $\omega \in \mathbb{R}$. Recalling the definition of $G_{+}$given in Remarks 2.2 $(h)$, 2.2( $(i)$ and the Popov criterion (2.11), which rewrites as

$$
q \omega \operatorname{Im}\left(G_{+}(i \omega)\right)-\operatorname{Re}\left(G_{+}(i \omega)\right)-\frac{1}{\beta}<0
$$

and using the series representation we arrive at

$$
-\frac{q}{\pi}-\frac{2}{\pi} \sum_{k=1}^{\infty}(-1)^{k} \frac{q \omega^{2}+k^{2}}{k^{4}+\omega^{2}}-\frac{1}{\beta}<0
$$

which is equivalent to 2.10). Notice that, by symmetry, it is enough to verify the criterion for $\omega \geq 0$, and since $G_{+}$has the explicit representation of Remarks 2.2(h) and 2.2(i), it is possible to use it to verify the validity of the condition.

Lemma 4.7. Let $y \in \mathrm{BC}((0, \infty), \mathbb{R})$ be a solution of the integral equation (2.1) with $u_{0} \in \mathrm{H}^{1}$. Then, for each $\beta \in\left(0, \beta_{0}\right)$, there is $q=q(\beta)>0$ such that

$$
R_{\beta, q(\beta)}(t) \leq 0 \text { for } t \geq 0
$$

where $\beta_{0} \approx 5.6655$ is the Hopf bifurcation value found in [11].

Proof. Define the functions

$$
A(\omega)=\frac{2}{\pi} \sum_{k=1}^{\infty}(-1)^{k+1} \frac{k^{2}}{k^{4}+\omega^{2}} \text { and } B(\omega)=-\frac{1}{\pi}+\frac{2}{\pi} \sum_{k=1}^{\infty}(-1)^{k+1} \frac{\omega^{2}}{k^{4}+\omega^{2}}
$$

so that condition (4.6) is satisfied if $\sup _{\omega \in \mathbb{R}}[A(\omega)+q B(\omega)]<\frac{1}{\beta}$ for some $q>0$. This is clearly only possible as long as

$$
\beta<\frac{1}{\inf _{q>0} \sup _{\omega \in \mathbb{R}}[A(\omega)+q B(\omega)]}=\sup _{q>0} \frac{1}{\sup _{\omega \in \mathbb{R}}[A(\omega)+q B(\omega)]}=: \sup _{q>0} M(q) .
$$

A plot of the (numerically computed) function $M$ shows that the critical value indeed coincides with $\beta_{0}$ from [11]. 


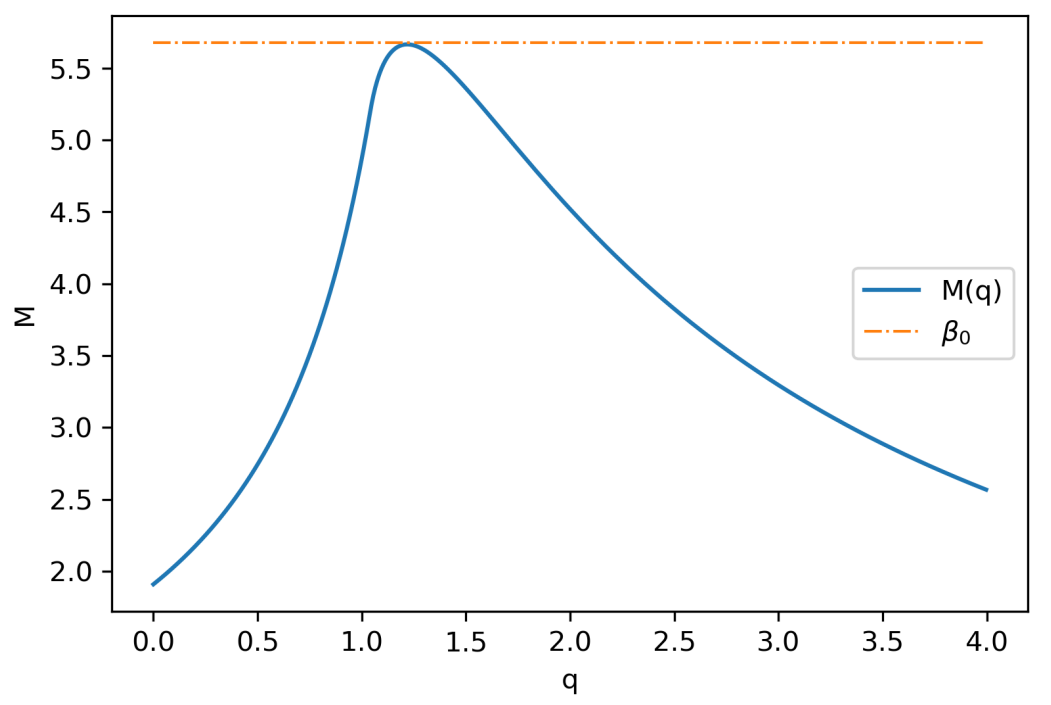

Figure 4. Graph of $\mathrm{M}(\mathrm{q})$ where its maximum value $\beta_{0}$ is determined numerically.

This result obviously only shows that we find a value numerically that coincides with $\beta_{0}$ in [1] up to the finite precision of the numerical method used for its calculation. To prove that the critical value of $\beta$ indeed equals the value determined in [11], we need additional considerations. First we describe a somewhat hidden but elementary symmetry between $\sin (z)$ and $\sinh (z)$ on the diagonal in the complex plane. This may be well-known in other contexts, but since we were not able to find an explicit reference we include it here for clarity.

Lemma 4.8. Set

$$
D:=\{z \in \mathbb{C} \mid \operatorname{Re}(z)=\operatorname{Im}(z)\}
$$

Then for $d \in D$ and $r \in \mathbb{R}$

$$
d \sin (r d) \in \mathbb{R} \Longleftrightarrow d \sinh (r d) \in \mathbb{R}
$$

and if the values are real, then

$$
d \sin (r d)=-d \sinh (r d)
$$

Proof. Note that for $d \in D$

$$
\frac{d}{i}=\bar{d} \text { and } i d=-\bar{d}
$$

We will also use that sinh is an odd function and that for $z \in \mathbb{C}$

$$
\sinh (\bar{z})=\overline{\sinh (z)} \text { and } \sin (z)=\frac{\sinh (i z)}{i} .
$$

Now assume $d \sin (r d) \in \mathbb{R}$ for $d \in D$ and $r \in \mathbb{R}$. Then using the above identities

$$
d \sin (r d)=d \frac{\sinh (i r d)}{i}=\bar{d} \sinh (-r \bar{d})=-\bar{d} \sinh (r \bar{d})=-\overline{d \sinh (r d)}
$$

which is the complex conjugate of $-d \sinh (r d)$ and thus proves the equivalence statement. Clearly, if the values are real then the calculation also shows that the claimed sign relationship must hold.

Now we can show that the critical $\beta$ determined from the Popov criterion coincides with $\beta_{0}$ obtained from the spectral analysis in [11]. 
Proposition 4.9. Let

$$
\Omega_{N y q}:=\left\{\omega>0 \mid \operatorname{Im}\left[G_{+}(i \omega)\right]=0\right\}
$$

and

$$
\Omega_{\text {Pop }}:=\left\{\omega>0 \mid \operatorname{Im}\left[\operatorname{Re}\left[G_{+}(i \omega)\right]+i \omega \operatorname{Im}\left[G_{+}(i \omega)\right]\right]=0\right\}
$$

Then

$$
\Omega_{P o p}=\Omega_{N y q}
$$

and

$$
\Omega_{\text {Pop }}=\{\omega>0 \mid \sqrt{i \omega} \sinh (\sqrt{i \omega} \pi) \in \mathbb{R}\}=\{\omega>0 \mid \sqrt{i \omega} \sin (\sqrt{i \omega} \pi) \in \mathbb{R}\} .
$$

Let

$$
\omega_{0}:=\min \left\{\omega>0 \mid \sqrt{i \omega} \sin (\sqrt{i \omega} \pi) \in \mathbb{R}^{+}\right\} .
$$

Then for the constant $\beta_{0}$ obtained in [1] determined by

$$
\beta_{0}=\sqrt{i \omega_{0}} \sin \left(\sqrt{i \omega_{0}} \pi\right)
$$

it holds that

$$
\beta_{0}=\sup _{q>0} M(q) .
$$

Proof. The identity $\Omega_{\mathrm{Pop}}=\Omega_{\mathrm{Nyq}}$ follows from the definition since $\omega=0$ is excluded from both sets. Hence both sets collect the positive values of $\omega$ at which $G_{+}(i \omega) \in \mathbb{R}$. By the explicit representation of $G_{+}$given in Remarks 2.2 and since for $z \neq 0$, clearly $z \in \mathbb{R} \Longleftrightarrow \frac{1}{z} \in \mathbb{R}$ we obtain that

$$
\Omega_{\text {Pop }}=\{\omega>0 \mid \sqrt{i \omega} \sinh (\sqrt{i \omega} \pi) \in \mathbb{R}\} .
$$

The second characterization of $\Omega_{\text {Pop }}$ that involves sin instead of sinh follows from Lemma 4.8 by setting $d=\sqrt{i \omega}$ and $r=\pi$. The reason why the condition

$$
\beta_{0}=\sqrt{i \omega_{0}} \sin \left(\sqrt{i \omega_{0}}\right)
$$

characterizes the constant $\beta_{0}$ is discussed in [11. In fact, for $\beta=\beta_{0}$ the generator of the linear semigroup possesses a complex conjugate pair of imaginary eigenvalues $\pm i \omega_{0}$ that gives rise to a Hopf bifurcation as the parameter crosses the value $\beta_{0}$. Finally, that $\beta_{0}=\sup _{q>0} M(q)$ follows from the fact the Popov curve intersects the real axis for $\omega=\omega_{0}$ and that this intersection is the most negative intersection point of the Popov curve with the real axis. This point is given by

$$
G_{+}\left(i \omega_{0}\right)=\frac{1}{\sqrt{i \omega_{0}} \sinh \left(\sqrt{i \omega_{0}} \pi\right)} .
$$

Since by (4.7) and Lemma 4.8

$$
\beta_{0}=\sqrt{i \omega_{0}} \sin \left(\sqrt{i \omega_{0}} \pi\right)=-\sqrt{i \omega_{0}} \sinh \left(\sqrt{i \omega_{0}} \pi\right)
$$

we obtain

$$
G_{+}\left(i \omega_{0}\right)=-\frac{1}{\beta_{0}}
$$

Therefore for any $\beta>\beta_{0}$ no half-plane in $\mathbb{C}$ exists that contains the real point $-\frac{1}{\beta}$ without intersecting the Popov curve. This geometric criterion is implicit in the Popov stability criterion, where the parameter $q>0$ varies the slope of the half-plane's boundary. Clearly, to be fully rigorous in this argument we would need to discuss the path and the asymptotics of the Popov curve in the complex plane in more detail. Here we are thus relying to some extent on the qualitative features of the Popov curve that was obtained numerically. 
The above result establishes a relationship between those values of $\beta \in \mathbb{R} \backslash\{0\}$ that produce a conjugate complex pair of purely imaginary eigenvalues of the operator

$$
A(\beta):=-\triangle+\beta \gamma_{0}^{\prime} \gamma_{\pi},
$$

studied in [12], and the real values of the Nyquist curve. In fact, the above result also implies that

$$
\{\beta \in \mathbb{R} \backslash\{0\} \mid \sigma(A(\beta)) \cap i \mathbb{R} \neq \emptyset\}=\left\{-\frac{1}{G_{+}(i \omega)} \mid \omega \in \mathbb{R} \backslash\{0\} \text { and }|\omega| \in \Omega_{\mathrm{Nyq}}\right\} .
$$

After these clarifications we can now formulate a lemma that exploits the possibility to control the sign of $R_{\beta, q(\beta)}$ for $\beta \in\left(0, \beta_{0}\right)$ to obtain a bound on $V_{\beta, q(\beta)}$.

Lemma 4.10. Let $y \in \mathrm{BC}((0, \infty), \mathbb{R})$ be a solution of the integral equation (2.1) with $u_{0} \in \mathrm{H}^{1}$. Let $\beta \in\left(0, \beta_{0}\right)$ and let $q=q(\beta)>0$ be such that $R_{\beta, q(\beta)}(t) \leq 0$ for $t \geq 0$. Then the function $V=V_{\beta, q(\beta)}$ defined in Lemma 4.4 satisfies

$$
V(t) \leq c<\infty \text { for } t \geq 0,
$$

with a constant $c$ independent of $t \geq 0$.

Proof. Since

$$
V(t)=\int_{0}^{t} g_{\beta}(y(\tau))\left[f(\tau)+q f^{\prime}(\tau)\right] d \tau+W_{2}(0),
$$

integration by parts yields

$$
\begin{aligned}
V(t)=\left(\int_{0}^{t} g_{\beta}(y(\tau)) d \tau\right)[f(t)+q & \left.f^{\prime}(t)\right]+ \\
& -\int_{0}^{t}\left[\int_{0}^{\tau} g_{\beta}(y(\sigma)) d \sigma\right]\left[f^{\prime}(\tau)+q f^{\prime \prime}(\tau)\right] d \tau+W_{2}(0) .
\end{aligned}
$$

Since $R_{\beta, q(\beta}(t) \leq 0$ and $W_{\beta, q(\beta)}(t) \geq 0$ for $t \geq 0$, then using 4.1) we conclude that, for $t \geq 0$,

$$
V(t) \geq V(t)+R_{\beta, q(\beta)}(t)=W_{\beta, q(\beta)} \geq W_{3}(t)=\frac{1}{2 \pi}\left(\int_{0}^{t} g_{\beta}(y(\tau)) d \tau\right)^{2} \geq 0 .
$$

It follows that

$$
\frac{1}{2 \pi}\left(\int_{0}^{t} g_{\beta}(y(\tau)) d \tau\right)^{2}-V(t) \leq 0, t \geq 0
$$

With

$$
K(t):=\sup _{\tau \in[0, t]}\left|\int_{0}^{\tau} g_{\beta}(y(\sigma)) d \sigma\right|,
$$

a constant $c>0$ can be found which is independent of $t \geq 0$ and such that

$$
\left|\left[f(t)+q f^{\prime}(t)\right] \int_{0}^{t} g_{\beta}(y(\tau)) d \tau\right| \leq c K(t)
$$

and

$$
\left|\int_{0}^{t}\left[\int_{0}^{\tau} g_{\beta}(y(\sigma)) d \sigma\right]\left[f^{\prime}(\tau)+q f^{\prime \prime}(\tau)\right] d \tau\right| \leq c K(t)
$$

for $t \geq 0$. This is a consequence of the boundedness of $y, f, f^{\prime}$, and $f^{\prime \prime}$ discussed in Remarks 2.2 . Therefore we infer from (4.8) that there is a $c>0$ such that

$$
0 \leq V(t) \leq c K(t)+W_{2}(0) \text { for } t \geq 0 \text {. }
$$

Defining

$$
E(t):=\int_{0}^{t} g_{\beta}(y(\tau)) d \tau, t \geq 0
$$


we infer from 4.9 that

$$
\frac{1}{2 \pi} E^{2}(t)-c \sup _{\tau \in[0, t]}|E(\tau)|-W_{2}(0) \leq 0, t \geq 0 .
$$

This finally implies that $E$ is bounded and therefore that $K$ is bounded, which concludes the proof by 4.10 .

Proposition 4.11. Let $\beta \in\left(0, \beta_{0}\right)$ and $\left.y \in \mathrm{BC}(0, \infty), \mathbb{R}\right)$ be a solution of $(2.1)$ with $u_{0} \in \mathrm{H}^{1}$. Then $y \in \operatorname{BUC}([0, \infty), \mathbb{R}))$.

Proof. Since $y$ solves (2.1), we have that

$$
y(t)=f(t)-\frac{1}{\pi} \int_{0}^{t} g_{\beta}(y(\tau)) d \tau+\int_{0}^{t} a_{s}(t-\tau) g_{\beta}(y(\tau)) d \tau,
$$

for $t \geq 0$ and where $f(t)=\left(e^{-t A_{N}} u_{0}\right)(\pi)$ is the forcing function induced by $u_{0}$. We proceed by verifying that each term is uniformly continuous. The first term is uniformly continuous by Remark 2.2 (b). For the second term note that in the proof of Lemma 4.10 it was shown that $E$ defined by $E(t)=\int_{0}^{t} g_{\beta}(y(\tau)) d \tau$ for $t \geq 0$ is bounded. It is clearly continuous by assumption (on $y$ ). Its derivative is given by $g_{\beta} \circ y$ which is a bounded function and hence implies that $E$ is uniformly Lipschitz. Finally, the last term can be written as a convolution

$$
\int_{0}^{t} a_{s}(t-\tau) g_{\beta}(y(\tau)) d \tau=\left(a_{s} *\left(g_{\beta} \circ y\right)\right)(t), t \geq 0 .
$$

Since $a_{s} \in \mathrm{L}^{1}([0, \infty))$ and $g_{\beta} \circ y \in \mathrm{L}^{\infty}([0, \infty))$, well-known results about the regularity of convolutions imply the stated uniform continuity (see e.g. [1] or [8]).

Before we can proceed with the proof of Proposition 4.1 we need the following elementary but important result.

Lemma 4.12. Let $y \in \operatorname{BUC}([0, \infty))$ and $\beta \in(0, \infty)$. Then the function

$$
g_{\beta}(y)\left[y-\frac{g_{\beta}(y)}{\beta}\right] \in \operatorname{BUC}([0, \infty)),
$$

and is positive unless $y=0$.

Proof. First observe that for $y \neq 0$

$$
g_{\beta}(y)\left[y-\frac{g_{\beta}(y)}{\beta}\right]=\frac{1}{\beta} \tanh (\beta y) \beta y\left[1-\frac{\tanh (\beta y)}{\beta y}\right],
$$

which shows that the function is positive unless $y=0$ since the functions $[z \rightarrow z \tanh (z)]$ and $\left[z \rightarrow 1-\frac{\tanh (z)}{z}\right]$, for $z=\beta y$, enjoy the same property and $\beta>0$. Next

$$
g_{\beta}(y)\left[y-\frac{g_{\beta}(y)}{\beta}\right]=\frac{1}{\beta} \tanh (z)[z-\tanh (z)]
$$

shows that the function is the product of bounded and unifornly continuous functions since $z$ is such and since tanh has a bounded derivative and is therefore globally Lipschitz continuous. Indeed this yields

$$
|\tanh (z(t))-\tanh (z(s))| \leq L|z(t)-z(s)|, t, s \in[0, \infty),
$$

so that the boundedness and uniform continuity of $z$ is inherited by $\tanh (z)$.

Having now collected all the required technical results in the previous lemmas we can finally give the proof of our main proposition on the integral equation (2.1). 
Proof of Proposition 4.1.

Since $\beta \in\left(0, \beta_{0}\right)$ and $y$ solves (2.1), we can apply Lemmas 4.7 and 4.10 to find $q(\beta)>0$ such that

$$
R_{\beta, q(\beta)}(t) \leq 0 \text { for } t \geq 0,
$$

and $c>0$ such that

$$
c \geq V_{\beta, q(\beta)}(t) \geq W_{\beta, q(\beta)}(t) \geq W_{1, \beta}(t) \geq 0,
$$

for $t \geq 0$, i.e. such that

$$
W_{1, \beta}(t)=\int_{0}^{t} g_{\beta}(y(\tau))\left[y(\tau)-\frac{g_{\beta}(y(\tau))}{\beta}\right] d \tau=: \int_{0}^{t} H(y(\tau)) d \tau \leq c<\infty,
$$

for $t \geq 0$. By Lemma 4.12 the function $H(y)$ is non-negative, only vanishes if $y=0$, and is uniformly continuous. Assume by contradiction that $y(t) \not \rightarrow 0$ as $t \rightarrow \infty$. Then, since $H(\xi)>0$ for $0 \neq \xi \in \mathbb{R}$, there is a sequence $\left(t_{m}\right)_{m \in \mathbb{N}}$ in $\mathbb{R}$ with $t_{m} \rightarrow \infty$ and a constant $c>0$ such that

$$
H\left(y\left(t_{m}\right)\right) \geq 2 c \text { for all } m \in \mathbb{N} .
$$

Since $H \circ y \in \operatorname{BUC}([0, \infty))$, a $\delta>0$ can be found such that

$$
H(y(t)) \geq c \text { for } t \in\left[t_{m}-\delta, t_{m}+\delta\right],
$$

and all $m \in \mathbb{N}$. It follows that

$$
W_{1}\left(t_{m}\right)=\int_{0}^{t_{m}} H(y(\tau)) d \tau \geq \sum_{k=1}^{m-1} \int_{t_{k}-\delta}^{t_{k}+\delta} H(y(\tau)) d \tau \geq(m-1) 2 \delta c,
$$

which contradicts the boundedness of $W_{1}$ on $[0, \infty)$ since $m$ can be chosen arbitrarily large.

\section{Global Stability and Convergence Results}

We finally state our main stability and convergence result by explicitly determining the global attractor of (1.1) for $\beta \in\left(0, \beta_{0}\right)$. Adopting the terminology in [17], we need to make the important distinction between the concept of the "minimal closed global B-attractor" $\mathcal{A}_{\beta}$ and the "minimal closed global attractor" $\hat{\mathcal{A}}_{\beta}$ of our semiflow $\left(\Phi_{\beta}, \mathrm{H}^{1}\right)$ generated by the problem $(1.1)$ for each $\beta \in(0, \infty)$. In general, it holds that the attractor $\hat{\mathcal{A}}_{\beta}$ defined by

$$
\hat{\mathcal{A}}_{\beta}:=\overline{\bigcup_{u \in H^{1}} \omega(u)}
$$

is a proper subset of the $\mathrm{B}$-attractor $\mathcal{A}_{\beta}$ that is defined, in a more implicit way, by

$$
\mathcal{A}_{\beta}:=\bigcup_{B \in \mathcal{B}} \omega(B)
$$

where $\mathcal{B}$ is the set of all bounded subsets of $H^{1}$.

By Theorem 2.3. in [17] the attractor $\hat{\mathcal{A}}_{\beta}$ coincides withe the set of all stationary points of the semiflow if a suitable Ljapunov function exists. Using this distinction between different attractor concepts we can now formulate our main result.

Theorem 5.1. For $\beta \in\left(0, \beta_{0}\right)$ the minimal closed global attractor $\hat{\mathcal{A}}_{\beta} \subset H^{1}$ of the continous semiflow $\left(\Phi_{\beta}, \mathrm{H}^{1}\right)$ generated by the nonlinear and nonlocal problem (1.1) is given by

$$
\hat{\mathcal{A}}_{\beta}=\{0\},
$$

where $\beta_{0} \approx 5.6655$ is the constant determined in [11.

For $\beta \in\left(0, \frac{4}{\pi}\right)$ the attractor $\hat{\mathcal{A}}_{\beta}$ and the B-attractor $\mathcal{A}_{\beta}$ coincide, i.e.

$$
\mathcal{A}_{\beta}=\hat{\mathcal{A}}_{\beta}=\{0\} \text {. }
$$


Proof. Let $u \in H^{1}$ arbitrary. Then by Lemma 2.1 the trace of the orbit $\gamma_{\pi}\left(\Phi_{\beta}(t, u)\right)$ solves the Volterra integral equation (2.1). By Proposition 4.1 the assumption $\beta \in\left(0, \beta_{0}\right)$ implies that as $t \rightarrow \infty$

$$
\gamma_{\pi}\left(\Phi_{\beta}(t, u)\right) \rightarrow 0 \text { in } \mathbb{R} .
$$

Finally, by Proposition 2.3 we conclude that

$$
\Phi_{\beta}(t, u) \rightarrow 0 \text { in } H^{1} \text { as } t \rightarrow \infty,
$$

which implies that the omega-limit set $\omega(u)=\{0\}$. Therefore $\hat{\mathcal{A}}_{\beta}=\{0\}$.

For $\beta \in\left(0, \frac{4}{\pi}\right)$ we have shown in Remark 1.2 that a Ljapunov function for the semiflow exists. By Theorem 2.3. in [17] the B-attractor $\mathcal{A}_{\beta}$ consists of complete trajectories connecting stationary states of the semiflow $\left(\Phi_{\beta}, H^{1}\right)$. Since we know that $\hat{\mathcal{A}}_{\beta}=\{0\}$ by the proof of the first statement and since non-trivial complete orbits connecting $\{0\}$ with itself are excluded by the existence of the Ljapunov function, we conclude that $\mathcal{A}_{\beta}=\hat{\mathcal{A}}_{\beta}=\{0\}$.

We conclude with some remarks on open problems and the extension of the results to a more general class of nonlinearities $g_{\beta}$, which so far we have assumed to be equal to $\tanh (\beta \cdot)$ for $\operatorname{simplicity.}$

Remarks 5.2. (a) Assume that for each $\beta \in(0, \infty)$ the function $g_{\beta}: \mathbb{R} \rightarrow \mathbb{R}$ has the following properties:

(i) $g_{\beta}$ is bounded and globally Lipschitz continous.

(ii) $g_{\beta}(0)=0$ and $g_{\beta}^{\prime}(0)=\beta$.

(iii) $g_{\beta}(w)\left(w-\frac{g_{\beta}(w)}{\beta}\right)>0$ for $w \neq 0$.

Then Theorem 5.1 remains true if $\tanh (\beta \cdot)$ in (1.1) is replaced by a different nonlinear function $g_{\beta}(\cdot)$ with the above properties. This follows from an inspection of the assumptions made on $g_{\beta}$. In particular, in the proof of Proposition 4.1 and in Remark 1.2.

(b) We conjecture that $\mathcal{A}_{\beta}=\hat{\mathcal{A}}_{\beta}=\{0\}$ for $\beta \in\left(0, \beta_{0}\right)$. Since we were not able to find a Ljapunov function for $\beta \geq \frac{4}{\pi}$, this natural conjecture may require a different proof that we don't have at this time.

(c) By the existence of a non-trivial periodic solution for $\beta \in\left(\beta_{0}, \beta_{0}+\varepsilon\right)$ for some $\varepsilon>0$ we know that $\{0\}$ is a proper subset of $\mathcal{A}_{\beta}$ for $\beta \in\left(\beta_{0}, \beta_{0}+\varepsilon\right)$. We conjecture that this is true for $\beta \in\left(\beta_{0}, \infty\right)$. (d) A proof of the two conjectures above would imply that $\left(0, \beta_{0}\right)$ is the maximal open set in $[0, \infty)$ where $\mathcal{A}_{\beta}=\hat{\mathcal{A}}_{\beta}=\{0\}$.

\section{REFERENCES}

[1] H. Amann. Linear and Quasilinear Parabolic Problems. Birkhäuser, Basel, 1995.

[2] J.M. Borwein and P.B. Borwein. Pi and the AGM. Canadian Mathematical Society Series of Monographs and Advanced Texts. John Wiley and Sons, 1987.

[3] P. Brunovský, P. Poláčik, and B. Sandstede. Convergence in general periodic parabolic equations in one space dimension. Nonlinear Analysis TMA, 18:209-215, 1992.

[4] R. Curtain and K. Morris. Transfer functions of distributed parameter systems: A tutorial. Automatica, 45(5):11011116, 2009.

[5] D. Daners and J. Glück. A Criterion for the Uniform Eventual Positivity of Operator Semigroups. Integral Equations and Operator Theory, 90(4):19 pp., 2018.

[6] D. Daners, J. Glück, and J.B. Kennedy. Eventually and asymptotically positive semigroups on Banach lattices. J. Differential Equations, 261(5):2607-2649, 2016.

[7] D.S. de Cássia, R. Broche, L.A.F. de Oliveira, and A.L. Pereira. Global attractor for an equation modelling a thermostat. Electron. J. Differential Equations, 2003(100):7 pp, 2003.

[8] G. B. Folland. Real analysis: modern techniques and their applications. Wiley, New York, 1984.

[9] J. A. Goldstein. Semigroups of Linear Operators and Applications. Oxford University Press, Oxford, 1985.

[10] M. Gonzalez, M. Gualdani, and J. Solà Morales. Instability and Bifurcation in a Trend Depending Pricing Model. Acta Aplicandae Mathematicae, 144(1):121-136, 2013. 
[11] P. Guidotti and S. Merino. Hopf bifurcation in a scalar reaction diffusion equation. Journal of Differential Equations, 140(1):209-222, 1997.

[12] P. Guidotti and S. Merino. Gradual loss of positivity and hidden invariant cones in a scalar heat equation. Differential and Integral Equations, 13(10-12):1551-1568, 2000.

[13] G. Infante and J. R. L. Webb. Loss of positivity in a nonlinear scalar heat equation. NoDEA Nonlinear Differential Equations Appl., 13:249-261, 2006.

[14] G. Infante and J. R. L. Webb. Nonlinear nonlocal boundary value problems and perturbed Hammerstein integral equations. Proc. Edinb. Math. Soc., 49:637-656, 2006.

[15] G. Kalna and S. McKee. The thermostat problem with a nonlocal nonlinear boudary condition. IMA J. Appl. Math., 69(5):437-462, 2004.

[16] H. K. Khalil. Nonlinear Systems. Prentice-Hall, Upper Saddle River, 2002.

[17] O.A. Ladyženskaja. Attractors for semigroups and evolution equations. Cambridge University Press, 1991.

[18] S Lang. Die Wärmeleitung auf dem Kreis und Thetafunktionen. Elemente der Mathematik, 51:17-27, 1996.

[19] S.D. Lawley. Blowup from Randomly Switching between Stable Boundary Conditions for the Heat Equation. Commun. Math. Sci., 16(4):1131-1154, 2018.

[20] R.K. Miller. Nonlinear Volterra Integral Equations. Mathematics Lecture Notes Series. W.A. Benjamin, Menlo Park, CA, 1971.

University of California, Irvine, Department of Mathematics, 340 Rowland Hall, Irvine, CA 926973875 , USA

Basler Kantonalbank, Brunngaesslein 3, CH-4002 Basel, Switzerland

E-mail address: gpatrick@math.uci.edu and sandro.merino@bkb.ch 